\title{
Evaluating Different Parameterizations for Mixed Layer Eddy Fluxes induced by Baroclinic Instability
}

\author{
NILS BRÜGGEMANN AND CARSTEN EDEN \\ University of Hamburg, Hamburg, Germany
}

(Manuscript received 28 October 2013, in final form 24 June 2014)

\begin{abstract}
In this study, the authors discuss two different parameterizations for the effect of mixed layer eddies, one based on ageostrophic linear stability analysis (ALS) and the other one based on a scaling of the potential energy release by eddies (PER). Both parameterizations contradict each other in two aspects. First, they predict different functional relationships between the magnitude of the eddy fluxes and the Richardson number $(\mathrm{Ri})$ related to the background state. Second, they also predict different vertical structure functions for the horizontal eddy fluxes. Numerical simulations for two different configurations and for a large range of different background conditions are used to evaluate the parameterizations. It turns out that PER is better suited to capture the Ri dependency of the magnitude of the eddy fluxes. On the other hand, the vertical structure of the meridional eddy fluxes predicted by ALS is more accurate than that of PER, while the vertical structure of the vertical eddy fluxes is well predicted by both parameterizations. Therefore, this study suggests the use of the magnitude of PER and the vertical structure functions of ALS for an improved parameterization of mixed layer eddy fluxes.
\end{abstract}

\section{Introduction}

High-resolution satellite altimetry and numerical simulations of the near-surface ocean show variability on scales much smaller than the typical Rossby radius of the ocean interior (Munk et al. 2000; Klein et al. 2008). The dynamics on these scales are often called submesoscales, and it is assumed that ageostrophic processes have to be taken into account to describe these phenomena. Submesoscale dynamics might predominantly occur within the ocean mixed layer, where the influence of diabatic effects like convective and wind-induced mixing leads to dynamic conditions that are not in quasigeostrophic balance anymore, although out of balance dynamics might also be met under certain circumstances in the ocean interior. Haine and Marshall (1998) investigate different mechanisms that play a role for the upper-ocean mixed layer dynamics. All these processes act to restratify the mixed layer and occur on length scales that are too small to be resolved by today's climate models. Consequently, Oschlies (2002) observed a bias in the mixed layer

Corresponding author address: Nils Brüggemann, University of Hamburg, Bundesstrae 53, Hamburg D-20146, Germany.

E-mail: nils.brueggemann@zmaw.de depth and the surface ocean heat flux in coarser but eddying ocean models, suggesting that these processes might also play an important role for, for example, biogeochemical cycles.

The discussion of ageostrophic instabilities by Stone (1966) shows that a key parameter to distinguish between different kinds of instabilities is the Richardson number $\mathrm{Ri}$ - the ratio between the vertical buoyancy gradient and the square of the vertical shear of horizontal velocity. Large Ri indicate a geostrophic balance, while dynamics with smaller $\mathrm{Ri}$ show larger ageostrophic effects. While baroclinic instabilities can occur for all Ri, symmetric instabilities are only met for $\mathrm{Ri}<1$, and Kelvin-Helmholtz instability are only met for $\mathrm{Ri}<0.25$. In addition, the growth rate of baroclinic and symmetric instabilities also depends on Ri. In typical, idealized model simulations of the spindown of ocean fronts (e.g., Haine and Marshall 1998; Boccaletti et al. 2007; Fox-Kemper et al. 2008; Bachman and FoxKemper 2013), symmetric instabilities start to restratify the ocean front if $\mathrm{Ri}<1$ and lead to stable conditions with respect to symmetric instabilities $(\mathrm{Ri} \geq$ 1). After this first phase of restratification and after the geostrophic adjustment, baroclinic instabilities set in and lead to a further spindown of the front caused by 
baroclinic eddy buoyancy fluxes. Ri representative of the background field can vary over a few orders of magnitude during this process, and geostrophic and also ageostrophic effects can be involved in the mixed layer restratification. Baroclinic instabilities occur in nearly every flow system with vertically sheared velocity and are therefore important to be parameterized if ocean models are not able to resolve them.

Stone (1966) and Molemaker et al. (2005) find that even for small Ri characteristic for the mixed layer, the dominating baroclinic instabilities are still geostrophically balanced to a large extent, although they might also come in concert with secondary unbalanced instabilities. This means that, if the mixed layer instabilities are predominantly in geostrophic balance, they might be well described by mesoscale eddy parameterizations designed for the interior of the ocean with large Ri (Green 1970; Gent and McWilliams 1990; Killworth 1997; Eden and Greatbatch 2008b; Eden 2011). On the other hand, there are also parameterizations developed for small $\mathrm{Ri}$ and thus ageostrophic baroclinic instabilities (Stone 1972a) and parameterizations especially developed for the mixed layer (FoxKemper et al. 2008). All these parameterizations need to somehow connect the magnitude of the eddy mixing to the mean state. Since the Richardson number Ri is an important measure for the characteristics of the mean state instabilities, it is not surprising that most parameterizations associate the magnitude of the eddy fluxes by some kind of functional relationship to Ri. Hence, these parameterizations can be distinguished by their dependency on Ri.

Fox-Kemper et al. (2008) discuss the difference between their parameterization and the one of Stone (1972b). They cast both parameterizations in the form of an eddy streamfunction that represents the adiabatic advective effect of the eddy fluxes and use numerical simulations of a restratifying density front to evaluate both parameterizations. They find that their parameterization is better suited to represent the magnitude of the eddy streamfunction than the parameterization of Stone (1972b). By parameterizing the eddy streamfunction only, Fox-Kemper et al. (2008) neglect diabatic effects, since they assume these to be small in their scenario of a restratifying density front. However, it is most likely that diabatic effects, for example, due to wind-induced turbulence or convective mixing, play a major role in the upper-ocean mixed layer (Tandon and Garrett 1996; Treguier et al. 1997). The aim of this study is to investigate how these diabatic effects modify the amplitude and vertical structure of the eddy fluxes. Furthermore, we evaluate to which extent parameterizations based on scalings of Stone (1972b) and
Fox-Kemper et al. (2008) are able to represent the eddy fluxes in a diabatic environment.

The parameterization based on the suggestions by Fox-Kemper et al. (2008) follows different physical arguments than that of Stone (1972a) [in the quasigeostrophic limit the latter is in fact very similar to the one by Killworth (1997) and Eden (2011)] and thus suggests a different functional relationship between $\mathrm{Ri}$ and the magnitude of the eddy fluxes. The aim of this study is to discuss differences in these two classes of parameterizations for mixed layer eddies. We consider two different setups. The first one is a diabatic, forced dissipative scenario in a statistically steady state. In this scenario, diabatic effects are implemented as an idealized forcing instead of resolving submesoscale and diabatic turbulence at the same time. The advantage of this idealized representation of the diabatic effects is that computational costs are low enough to allow performing simulations for a wide range of parameters. The second scenario consists of a restratifying density front where diabatic effects are by far less strong than in the forced dissipative scenario. The latter scenario is the same that is also used by Fox-Kemper et al. (2008) and Bachman and Fox-Kemper (2013).

By comparing the different $\mathrm{Ri}$ dependencies of the parameterizations with the diagnosed dependency in a numerical model, we aim to clarify which Ri dependency is more appropriate to parameterize the eddy fluxes. Since both classes of parameterizations differ also with respect to the vertical structure of the meridional eddy fluxes, we furthermore compare the vertical profiles of the eddy fluxes from the numerical simulations and that of the parameterizations. All these investigations are performed for the two different scenarios, the equilibrated forced dissipative scenario and the spindown scenario of a density front, and over a wide range of values for $\mathrm{Ri}$.

In the following, we will introduce the parameterizations of Stone (1972a) and Fox-Kemper et al. (2008) in section 2, where we also discuss how our variant of the latter differs from the original one by Fox-Kemper et al. (2008). After that, numerical simulations for the two different scenarios are considered to evaluate these parameterizations. First, we discuss the forced dissipative scenario of a baroclinically unstable flow, where restratification is prevented by temperature restoring. The description of the setup and a discussion of the results can be found in section 3. Second, we discuss in section 4 setup and results of a scenario that consists of a baroclinically unstable and restratifying density front. Last, section 5 provides a discussion of the obtained results and their implications for parameterizing mixed layer eddy fluxes in numerical ocean models. 


\section{Parameterizations for mixed layer eddy fluxes}

To discuss the influence of mixed layer eddy fluxes on the mean buoyancy budget, we average the equation for buoyancy $b$ :

$$
\partial_{t} \bar{b}+\nabla \cdot \overline{\mathbf{u}} \bar{b}+\nabla \cdot \overline{\mathbf{u}^{\prime} b^{\prime}}=\bar{D} .
$$

In Eq. (1), the local change of mean buoyancy $\bar{b}$ is given by the advection of mean buoyancy by the mean velocity $\overline{\mathbf{u}}$, the divergence of the eddy fluxes $\overline{\mathbf{u}^{\prime} b^{\prime}}$, and diabatic processes denoted by $D$. While the mean flux $\overline{\mathbf{u}} \bar{b}$ is simulated in a numerical ocean model, the eddy flux $\overline{\mathbf{u}^{\prime} b^{\prime}}$ needs to be parameterized. For simplicity, we consider zonal averages, ${ }^{1}$ that is, $\overline{(~)}$ denotes a zonal average and ()$^{\prime}$ denotes the deviations from that average. Equation (1) contains only velocity components and gradients in the meridional-vertical plane.

As described in the following, the structure and strength of the eddy fluxes depends on the background state of the flow. A nondimensionalization of the NavierStokes equations shows that basically three parameters determine the inviscid adiabatic equations, namely, the Richardson number Ri, the Rossby number Ro, and the aspect ratio $\delta$ (see appendix A). Therefore, it is convenient to derive parameterizations for the eddy fluxes in dependency on these parameters.

\section{a. A parameterization based on linear stability analysis}

The concept of using linear stability analysis to determine eddy fluxes is based on the following ideas (see, e.g., Green 1970; Stone 1972a; Killworth 1997; Eden 2011): As long as perturbations to a mean state are small, all perturbation variables can be obtained by solving a linearized set of equations for waves. From the solution for these perturbation variables, the eddy fluxes can be calculated from the correlations between velocity and buoyancy perturbation. If the frequency for certain wave modes becomes complex, there is an exponential increase or decay of these waves. The fastest growing mode is expected to dominate over the others, and therefore this fastest growing mode is assumed to be responsible for the eddy mixing.

As soon as the exponentially growing perturbations reach the same magnitude as the background state, the linearization of the equation is certainly no longer

\footnotetext{
${ }^{1}$ Note that a zonal average implies a strongly anisotropic averaging operation. Therefore, a zonal average in general differs from the mean state inferred by a three-dimensional model. This has to be considered if the results obtained in this study are used to implement the parameterization in a three-dimensional model.
}

a good approximation, and nonlinear effects become important. However, as long as there is no significant energy cascade transporting energy to different scales, one might assume that the eddies retain their initial scales and structure and that it is possible to infer on the eddy fluxes by the linear solutions. Nevertheless, it remains to find a proper scaling for the magnitude of the eddy fluxes at the end of their exponential growth in the fully turbulent regime. We note that the assumption of a small effect of an energy cascade is a major drawback of approaches, which parameterize nonlinear eddy fluxes by linear theory. However, such approaches are successfully used in many studies (e.g., Stone 1972b; Killworth 1997; Eden 2011) to parameterize geostrophic adiabatic turbulent eddy fluxes. We thus aim to test if it also yields reasonable results in the ageostrophic and diabatic regime investigated in this study.

Stone (1972a) argues that the exponential growth of the wave is stopped as soon as nonlinear effects become important. Thus, the perturbation velocity $v^{\prime}$ takes the same order of magnitude as the background velocity, and $v^{\prime} \propto M^{2} H / f$ yields the scaling for the eigenvectors, where $M^{2}$ is the meridional buoyancy gradient, $H$ is the water depth, and $f$ is the Coriolis parameter. A different idea to scale the amplitude of the eigenvectors is suggested by Killworth (1997) who suggests using the time and length scale of the fastest growing mode to scale the meridional velocity component $v^{\prime}$. For quasigeostrophic conditions (i.e., large $\mathrm{Ri}$ ), constant meridional and vertical buoyancy gradients $M^{2}$ and $N^{2}$, respectively, and the vanishing planetary vorticity gradient, the growth rate $\sigma_{\max }$ of the fastest growing mode and the corresponding wavenumber $k_{\max }$ can be derived as follows (Eady 1949):

$$
k_{\max } \approx 1.6 L_{r}^{-1}, \quad \sigma_{\max } \approx 0.3 \frac{f}{\sqrt{\mathrm{Ri}}},
$$

where $L_{r}=N H / f$ denotes the Rossby radius, and $\mathrm{Ri}=$ $N^{2} f^{2} / M^{4}$ denotes the Richardson number for a flow in geostrophic balance with depth $H$. Applying the scaling of Killworth (1997) then yields $v^{\prime}=\sigma_{\max } / k_{\max } \approx$ $0.25 M^{2} H / f$ and therefore basically the same scaling as suggested by Stone (1972a).

Following the ideas of Stone (1972a) and Killworth (1997), it is possible to calculate exact expressions for the eddy fluxes of the Eady (1949) model as detailed in appendix B:

$$
\frac{\overline{v^{\prime} b^{\prime}}}{H^{2} f^{3}}=-1.9 C_{E} \sqrt{\mathrm{Ri}} \alpha^{3}, \quad \frac{\overline{w^{\prime} b^{\prime}}}{H^{2} f^{3}}=C_{E} \frac{1}{\sqrt{\mathrm{Ri}}} \mu_{E}(z) \alpha^{2},
$$

where $C_{E}$ denotes a tuning constant of order one, and $\alpha=\mathrm{Ro} / \delta$ is the ratio between the Rossby number 

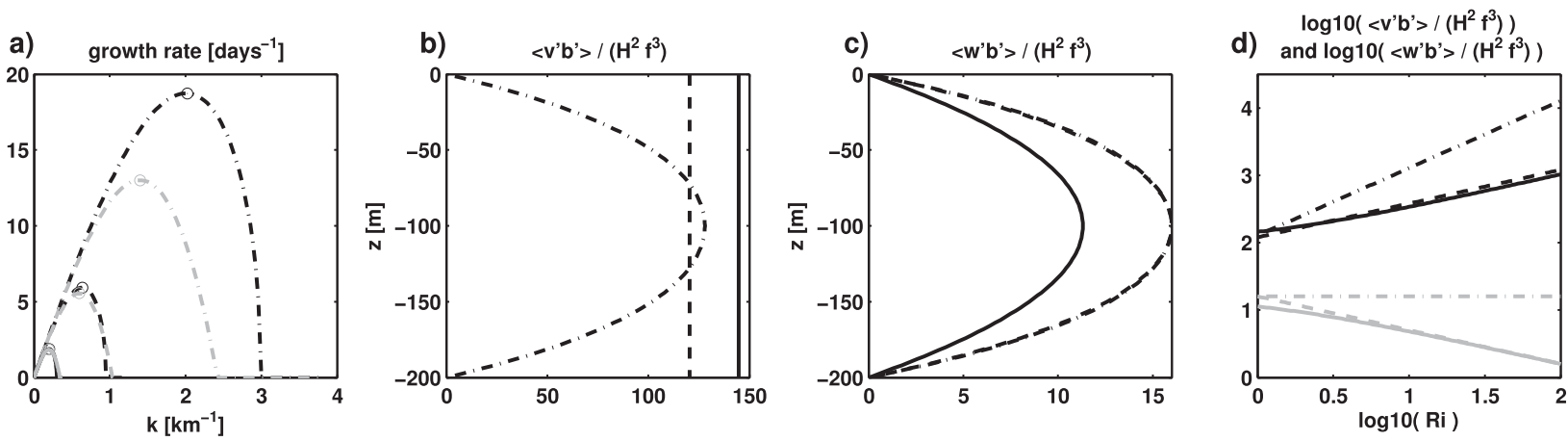

FIG. 1. (a) Growth rate inferred from linear stability analysis for $\alpha=4$ as a function of the along-stream wavenumber $k$ for the solution by Eady (black) and Stone (gray) for $\mathrm{Ri}=1$ (dashed-dotted), $\mathrm{Ri}=10$ (dashed), and $\mathrm{Ri}=100$ (solid). Maximum growth rates $\sigma_{\text {max }}$ at wavenumbers $k_{\max }$ are indicated by circles. (b)-(d) Eddy fluxes of the parameterizations based on Eq. (3) (dashed), Eq. (6) (solid), and Eq. (9) (dashed-dotted). (b) and (c) show the vertical structure function of $\overline{v^{\prime} b^{\prime}}$ and $\overline{w^{\prime} b^{\prime}}$, respectively, for $\mathrm{Ri}=1$ and $\alpha=4$ [note that the dashed line in (c) is superimposed on the dashed-dotted line]. (d) shows the amplitude of the eddy fluxes $\overline{v^{\prime} b^{\prime}}$ (black) and $\overline{w^{\prime} b^{\prime}}$ (gray) as a function of Ri for $\alpha=4$.

$\mathrm{Ro}=U /(f L)$ and the aspect ratio $\delta=H / L$ for a flow with velocity scale $U$ and horizontal and vertical length scales $L$ and $H$, respectively. While $\overline{v^{\prime} b^{\prime}}$ is vertically constant, the vertical dependency of $\overline{w^{\prime} b^{\prime}}$ is denoted by $\mu_{E}(z)$, which has a maximum of one at $z=-H / 2$ and is given by

$\mu_{E}(z)=\frac{\cosh \left[k_{\max } L_{r}\left(\frac{2 z}{H}+1\right)\right]-\cosh \left(k_{\max } L_{r}\right)}{1-\cosh \left(k_{\max } L_{r}\right)}$.

This kind of closure was already successfully implemented and evaluated in numerical ocean models in Killworth (1997), and Eden (2011, 2012) for general profiles of $N^{2}$ and $M^{2}$ and including also the planetary vorticity gradient.

Within the mixed layer, especially at fronts, strong vertical shears and weak stratifications are likely to occur such that $\mathrm{Ri}$ becomes small and ageostrophic processes have to be taken into account. For the ageostrophic equations, it is no longer possible to find analytical solutions as for the quasigeostrophic Eady problem even for constant $N^{2}$ and $M^{2}$ and vanishing planetary vorticity gradient. Stone (1966) derives approximate solutions for the ageostrophic, linearized equations in order to extent the theory of Eady (1949) for small Ri. For the fastest growth rate $\sigma_{\max }$ and the corresponding wavelength $k_{\max }$, Stone (1966) obtains

$$
k_{\max }=\sqrt{\frac{\mathrm{Ri}}{1+\mathrm{Ri}}} \sqrt{\frac{5}{2}} L_{r}^{-1}, \quad \sigma_{\max }=\sqrt{\frac{\mathrm{Ri}}{1+\mathrm{Ri}}} \sqrt{\frac{5}{54}} \frac{f}{\sqrt{\mathrm{Ri}}} .
$$

The only difference between these results and the ones from Eady (1949) is an additional factor $\sqrt{\mathrm{Ri} /(1+\mathrm{Ri})}$ that approaches one for large Ri. Figure $1 \mathrm{a}$ indicates that there is hardly any disagreement between the solutions of Eady (1949) and Stone (1966) for large Ri, while for smaller $\mathrm{Ri}$ the factor $\sqrt{\mathrm{Ri} /(1+\mathrm{Ri})}$ becomes more and more important (see, e.g., dashed-dotted lines in Fig. 1a for $\mathrm{Ri}=1)$.

As before, it is possible to derive the eddy fluxes by linear stability analysis up to a proportionality constant from the correlations of the eigenvectors for $v^{\prime}, w^{\prime}$, and $b^{\prime}$. If we use the same scaling as suggested by Killworth (1997), namely, assuming $\sqrt{\overline{v^{\prime} \boldsymbol{v}^{\prime}}} \propto \sigma_{\max } / k_{\max }$, we obtain

$$
\frac{\overline{v^{\prime} b^{\prime}}}{H^{2} f^{3}}=-\frac{8}{5} C_{S} \sqrt{1+\mathrm{Ri}} \alpha^{3}, \quad \overline{w^{\prime} b^{\prime}}=C_{S} \frac{1}{H^{2} f^{3}} \mu_{S}(z) \alpha^{2},
$$

where $C_{S}$ denotes another tuning parameter of order one. Formally, these equations are valid only in the longwave limit as noted by Stone (1972b). We use this approximate form and compare the eddy fluxes obtained by Eq. (6) with numerical solutions of the eigenvalue problem below and find good agreement. As in Eady's solution and depicted in Fig. 1b, there is no vertical dependency of $\overline{v^{\prime} b^{\prime}}$ in Stone's solution. The vertical structure of $\overline{w^{\prime} b^{\prime}}$ denoted by $\mu_{S}(z)$ is derived by Stone (1972b) as

$$
\mu_{S}(z)=-4 \frac{z}{H}\left(\frac{z}{H}+1\right)
$$

and therefore only marginally deviates from the hyperbolic structure of $\overline{w^{\prime} b^{\prime}}$ obtained for the Eady problem (see Fig. 1c). The parameterization based on the approximate analytic solution of the linear stability analysis is referred to as ALS in the following.

Although the solution of Stone (1972a) accounts for ageostrophic effects, it is quite similar to the one obtained by Eady (1949) for the quasigeostrophic limit of large Ri. Figure 1d shows the maximum of the eddy 
fluxes $\overline{v^{\prime} b^{\prime}}$ (solid curves) and $\overline{w^{\prime} b^{\prime}}$ (dashed curves) for both parameterizations over a large range of values for $\mathrm{Ri}$. The only differences for $\mathrm{Ri}=O(1)$ between Eady's and Stone's solution are due to the additional factor $\sqrt{\mathrm{Ri} /(1+\mathrm{Ri})}$ in Stone's solution. For large Ri, this factor approaches one and Stone's solution becomes identical to the one of Eady (1949).

\section{b. A parameterization based on potential energy release}

We also discuss a parameterization following arguments from Fox-Kemper et al. (2008), who derive a parameterization for the spindown of a baroclinic density front with typical mixed layer conditions by scaling the magnitude of the potential energy release. The key assumption for this class of closure is that the potential energy release $\left(\Delta \mathrm{PE}=g / \rho_{0} \Delta \rho \Delta z\right)$ is achieved by the vertical eddy flux. Thus, it is assumed that $\Delta \mathrm{PE} / \Delta t \propto-\Delta z\left(\Delta y M^{2}+\Delta z N^{2}\right) / \Delta t=-\overline{w^{\prime} b^{\prime}}$, where $\Delta z$ and $\Delta y$ denote the vertical and horizontal eddy length scales, respectively, and $\Delta t$ is the eddy time scale. To infer these eddy length and time scales, the following assumptions are made:

(i) The eddy time scale is an advective time scale $\Delta t \propto$ $\Delta y / U_{e}$, where $U_{e}$ denotes the eddy velocity.

(ii) The eddy velocity is set to be proportional to the thermal wind velocity $U_{e} \propto\left(M^{2} / f\right) H$.

(iii) The eddy depth scale $\Delta z$ is assumed to be proportional to the mixed layer depth $H$.

(iv) The eddy fluxes are along surfaces inclined by half of the isopycnal slope, that is, $\Delta z / \Delta y=-0.5 \mathrm{M}^{2} / N^{2}$ as can be inferred from parcel theory (Haine and Marshall 1998).

The second assumption is also made by Stone (1972a). Bachman and Fox-Kemper (2013) argue that this assumption is problematic and find reduced deviations between parameterized and diagnosed eddy fluxes, if the diagnosed eddy velocity is used instead of the thermal wind velocity. We also find this result here (not shown) but we make no attempt to improve this scaling to keep the closure as simple as possible. The assumptions iii and iv do not contradict the results of the linear stability analysis. Therefore, the parameterizations given by Eq. (6) and by Eq. (9) below differ only with respect to the first assumption: ${ }^{2}$ while this assumption of FoxKemper et al. (2008) leads to a time scale $T_{e}=\mathrm{Ri} / f$ and a corresponding length scale $L_{e}=U_{e} / T_{e}=\operatorname{Ri} \alpha H$, the scaling by Stone (1972a) as well as that by Killworth

\footnotetext{
${ }^{2}$ Note that assumption iv only corresponds to Stone (1972a) at the steering level.
}

(1997) suggest $T_{e}=\sqrt{1+\mathrm{Ri}} / f$ and correspondingly $L_{e}=$ $\sqrt{1+\operatorname{Ri}} \alpha H$.

The assumptions discussed so far yield only the magnitude of the eddy fluxes. Fox-Kemper et al. (2008) use the vertical structure $\mu(z)$ obtained from the linear stability analysis for $\overline{w^{\prime} b^{\prime}}$. In fact, Fox-Kemper et al. (2008) use a higher-order solution in the zonal wavenumber for the linear stability analysis than that of Stone (1972b) and apply to this solution the large $\mathrm{Ri}$ limit:

$$
\mu_{F}(z)=-4 \frac{z}{H}\left(\frac{z}{H}+1\right)\left[1+\frac{5}{21}\left(\frac{2 z}{H}+1\right)^{2}\right] .
$$

To obtain $\overline{v^{\prime} b^{\prime}}$, Fox-Kemper et al. (2008) assume $\overline{v^{\prime} b^{\prime}}=$ $-2 \overline{w^{\prime} b^{\prime}} N^{2} / M^{2}$ (corresponding to assumption iv). We thus obtain

$\frac{\overline{v^{\prime} b^{\prime}}}{H^{2} f^{3}}=-2 C_{F} \mu_{F}(z) \operatorname{Ri} \alpha^{3}, \quad \frac{\overline{w^{\prime} b^{\prime}}}{H^{2} f^{3}}=C_{F} \mu_{F}(z) \alpha^{2}$,

where $C_{F}$ is another tuning parameter of order one.

We stress that Fox-Kemper et al. (2008) cast their parameterization not as in Eq. (9) but as a streamfunction for an eddy-driven velocity that they define as $\Psi^{\mathrm{HS}}=-\overline{w^{\prime} b^{\prime}} / \partial_{y} \bar{b}$ in accordance with the definition of Held and Schneider (1999). To fully represent the eddy buoyancy fluxes, a residual eddy flux has to be added to the effect of the eddy-driven advection, which is for the case of $\Psi^{\mathrm{HS}}$ entirely in horizontal direction [other choices are possible; see, e.g., Plumb and Ferrari (2005) or Eden and Greatbatch (2008a)]. However, Fox-Kemper et al. (2008) argue that these residual eddy fluxes are small in comparison to horizontal numerical diffusion normally implemented in coarse ocean models. Therefore, they suggest only using $\Psi^{\mathrm{HS}}$ calculated by Eq. (9) to represent the eddy fluxes. Neglecting the residual eddy fluxes, the parameterization of Fox-Kemper et al. (2008) becomes thus fully adiabatic. In contrast to Fox-Kemper et al. (2008), we will use here the horizontal and the vertical eddy fluxes as given by Eq. (9), which makes the closure Eq. (9) diabatic, in order to allow the closure to also represent the diabatic effect of the eddy fluxes. Therefore, the closure given by $\overline{v^{\prime} b^{\prime}}$ and $\overline{w^{\prime} b^{\prime}}$ of Eq. (9) will be referred to as the parameterization based on potential energy release (PER) [and not as the parameterization by Fox-Kemper et al. (2008)].

\section{c. Comparison of the parameterizations}

There are two principal differences between PER given by Eq. (9) and ALS given by Eq. (6). The first one is a different dependency of the eddy fluxes on Ri resulting from assumption one of the last section. This means that even if both solutions are accurately tuned 
for a certain $\mathrm{Ri}$, there might be a difference in the magnitude of the eddy fluxes for different Ri. Figure 1d shows the maxima of the eddy fluxes $\overline{v^{\prime} b^{\prime}}$ and $\overline{w^{\prime} b^{\prime}}$ from Eq. (9) together with the eddy fluxes of the quasigeostrophic linear stability problem Eq. (3) and the ageostrophic problem Eq. (6). Here, the tuning constants are chosen in the way that both parameterizations agree best for $\mathrm{Ri}=1$. For $\mathrm{Ri}=100$, however, the difference between both parameterizations is roughly one order of magnitude. Choosing different tuning coefficients will shift the curves parallel to the vertical axis in the double logarithmic plot, but there will always remain a large difference for certain $\mathrm{Ri}$.

The second difference concerns the vertical structure of the horizontal eddy flux $\overline{v^{\prime} b^{\prime}}$. Because for PER, $\overline{w^{\prime} b^{\prime}} / \overline{v^{\prime} b^{\prime}}=0.5 M^{2} / N^{2}$ is assumed everywhere and not only at the steering level; $\overline{v^{\prime} b^{\prime}}$ has a parabolic vertical structure that vanishes at the surface and the mixed layer base. In contrast, linear stability analysis suggests a constant horizontal flux throughout the whole mixed layer (see Fig. 1b).

The differences between both parameterizations become more illustrative if we consider for the moment a downgradient closure for the horizontal eddy fluxes and the ratio of the horizontal and the vertical eddy fluxes:

$$
\overline{v^{\prime} b^{\prime}}=-K \partial_{y} \bar{b}, \quad \overline{w^{\prime} b^{\prime}}=\gamma \overline{v^{\prime} b^{\prime}}
$$

with the lateral diffusivity $K=-\overline{v^{\prime} b^{\prime}} / \partial_{y} \bar{b}$ and the eddy flux ratio $\gamma=\overline{w^{\prime} b^{\prime}} / \overline{v^{\prime} b^{\prime}}$. To parameterize $K$, it is common to use mixing length theory and to express $K$ as the product of a typical eddy velocity $U_{e}$ and a length scale $L_{e}$ :

$$
K \propto U_{e} L_{e} .
$$

Both parameterizations, PER and ALS, assume that the eddy velocity scale is proportional to the thermal wind velocity and that the eddy fluxes are along surfaces inclined by $s / 2$ at the steering level, where $s=-M^{2} / N^{2}$ denotes the isopycnal slope. Thus, the difference between both parameterizations concerning the amplitude of the eddy fluxes can be identified from a different choice of the eddy length scale. Linear stability analysis predicts $L_{e}=\sqrt{(\mathrm{Ri}+1) / \mathrm{Ri}} L_{r}$ and therefore a length scale essentially given by the Rossby radius $L_{r}$ with only small deviations for $\mathrm{Ri}=O(1)$. In contrast, PER implies $L_{e}=H N^{2} /\left|M^{2}\right|=\sqrt{\mathrm{Ri}} L_{r}$ as a characteristic eddy length scale, that is, a length scale that deviates from the parameterization based on linear stability analysis.

One might now construct the amplitude of the eddy fluxes $\overline{v^{\prime} b^{\prime}}$ and $\overline{w^{\prime} b^{\prime}}$ with Eqs. (10) and (11). By assuming $U_{e}=M^{2} H / f, \gamma \approx 1 / 2 s$ and $L_{0}=\sqrt{\operatorname{Ri}} L_{r}$ or $L_{0}=$ $\sqrt{(\mathrm{Ri}+1) / \mathrm{Ri}} L_{r}$, we obtain either PER or ALS, respectively. Instead of composing the lateral diffusivity by a velocity and a length scale, it would also be possible to compose it by a length and a time scale $T_{e}$. If we assume the latter to be $T_{e}=L_{e} / U_{e}$, we obtain $T_{e}=\sqrt{\mathrm{Ri}+1} / f$ for ALS and $T_{e}=\mathrm{Ri} / f$ for PER. Both time scales as well as the length scales differ.

In an adiabatic steady state, the diapycnal component of the eddy fluxes will vanish (except for a possible rotational component), and $\gamma$ will be equal to the isopycnal slope as assumed by, for example, Gent et al. (1995), Killworth (1997), and Eden (2011). In the presence of small-scale diabatic processes, however, there will be a net diapycnal transport of the eddies (Tandon and Garrett 1996; Eden and Greatbatch 2008a). Because the mixed layer is predestined to those diabatic processes (Tandon and Garrett 1996; Treguier et al. 1997), it is reasonable to expect nonvanishing diapycnal eddy fluxes and diapycnal diffusivities also in equilibrated scenarios for the mixed layer. In fact, we explicitly apply diabatic conditions to prevent the buoyancy front from slumping down in our equilibrated scenarios. Therefore, it seems more promising to assume $\gamma<s$ than $\gamma=s$ for our equilibrated scenario but probably also for the real mixed layer.

If the system is not steady, as is the case for a restratifying density front, $\gamma$ is likely to be smaller than the isopycnal slope even if the flow is completely adiabatic. Green (1970) and Stone (1972a) suggest that $\gamma$ takes values at the steering level close to $0.5 s$. Similarly, FoxKemper et al. (2008) suggest $\gamma=0.5 \mathrm{~s}$ for the whole vertical profiles of $\overline{v^{\prime} b^{\prime}}$ and $\overline{w^{\prime} b^{\prime}}$ and not only at the steering level. As detailed below, we also expect diabatic effects to occur in our spindown scenario due to numerical diffusion. Therefore, we cannot expect a completely adiabatic restratification process in the spindown scenario, although the diabatic effects are likely to be much smaller than in the equilibrated scenario. However, as mentioned above, a diabatic scenario is probably not unrealistic with respect to the ocean mixed layer, where diabatic processes will certainly accompany the restratification by baroclinic instabilities.

These considerations bring us to the following questions, which we aim to answer by the diagnosis of numerical model results:

(i) Which dependency of $\overline{v^{\prime} b^{\prime}}$ and $\overline{w^{\prime} b^{\prime}}$ on Ri is more appropriate, the one based on ALS given by Eq. (6) or the one based on PER given by Eq. (9)?

(ii) What is the vertical structure of the horizontal eddy fluxes $\overline{v^{\prime} b^{\prime}}$, a constant profile as suggested by ALS or a parabolic-shaped structure as suggested by PER?

(iii) Are there qualitative differences of the parameter dependency of the eddy fluxes in a spindown 
scenario in comparison to an equilibrated, diabatic, forced dissipative scenario?

\section{Baroclinic instabilities in a forced dissipative scenario}

\section{a. Numerical simulations}

To simulate mixed layer instabilities, we use the Massachusetts Institute of Technology general circulation model (MITgcm; Marshall et al. 1997). The configuration resembles that of Eady (1949) and Stone (1966). Our model domain consists of a reentrant channel with periodic boundary conditions at zonal boundaries and solid walls at meridional boundaries. For simplicity, we use temperature as the only active tracer and a linear equation of state; thus, temperature and buoyancy are equivalent. To test the parameterization for different $\mathrm{Ri}$ and $\alpha$, we vary vertical and meridional buoyancy gradients $N_{0}^{2}$ and $M_{0}^{2}$ to obtain specific values for $\mathrm{Ri}_{0}$ and $\alpha_{0}$ in accordance with

$$
\mathrm{Ri}_{0}=\frac{N_{0}^{2} f^{2}}{M_{0}^{4}}, \quad \alpha_{0}=\frac{M_{0}^{2}}{f^{2}} .
$$

The initial temperature $T_{0}$ is chosen to resemble the vertical and meridional stratification $N_{0}^{2}$ and $M_{0}^{2}$. The initial velocity is chosen to be in thermal wind balance with the initial temperature. A uniform depth of $H=200 \mathrm{~m}$ and Coriolis parameter of $f=7 \times 10^{-5} \mathrm{~s}^{-1}$ are applied throughout all simulations. The domain width is chosen equal in the zonal and meridional direction and allows for four wave lengths of the most unstable wave to fit in the domain. Since we use a resolution of 120 grid points in the horizontal, the horizontal resolution varies in dependency on $\mathrm{Ri}_{0}$ and $\alpha_{0}$ (see Table 1 ). In contrast, we use a constant vertical resolution of 40 layers and 5-m depth for each experiment (results from sensitivity experiments for different spatial resolutions are described in section 3c).

The described setup is baroclinically unstable, and small perturbations that we add to $T_{0}$ exponentially grow to eddies that drain their kinetic energy out of the mean state by relaxing the temperature front. To obtain an equilibrated scenario, we apply a temperature forcing that counteracts the restratification effect. This forcing is achieved by a restoring of the zonal-mean temperature $\bar{T}$ to the target temperature $T_{0}$ that is identical to the initial condition with an inverse time scale $\lambda_{\bar{T}}=2 \sigma_{\max }$, where $\sigma_{\max }$ is the growth rate determined by Eq. (5) with $\mathrm{Ri}_{0}$ and $\alpha_{0}$. This means we add a tendency term $\lambda_{\bar{T}}\left(T_{0}-\bar{T}\right)$ to the temperature conservation equation in the model. This kind of restoring has the advantage that the zonal-mean front is preserved without damping
TABLE 1. Overview of model parameters.

\begin{tabular}{lll}
\hline \multicolumn{1}{c}{ Symbol } & \multicolumn{1}{c}{ Meaning } & \multicolumn{1}{c}{ Value } \\
\hline (nx, ny, nz) & Number of grid points & $(120,120,40)$ \\
& $\quad$ in $x, y, z$ directions \\
$H$ & Depth of the basin & $200 \mathrm{~m}$ \\
$f$ & Coriolis parameter & $7 \times 10^{-5} \mathrm{~s}^{-1}$ \\
$U_{0}$ & Zonal velocity & $\alpha_{0} H f$ \\
$M_{0}$ & Meridional buoyancy & $\sqrt{\alpha_{0}} f$ \\
& $\quad$ gradient & \\
$N_{0}$ & Vertical buoyancy gradient & $\alpha_{0} \sqrt{\mathrm{Ri}_{0}} f$ \\
$k_{\max }$ & Wavenumber of fastest & $\sqrt{5 / 2} /\left(\sqrt{1+\mathrm{Ri}_{0}} \alpha_{0} H\right)$ \\
\multicolumn{4}{c}{$\quad$ growing wave } \\
$\sigma_{\max }$ & Growth rate of fastest & $\sqrt{5 / 54} / \sqrt{1+\mathrm{Ri}_{0}} f$ \\
$\Delta x$ & $\quad$ growing wave & \\
$A_{4}$ & Horizontal resolution & $8 \pi / k_{\max } / n x$ \\
& Biharmonic horizontal & $U_{0} d x^{3} / 20$ \\
$A_{v}$ & $\quad$ viscosity & \\
$\lambda_{u}$ & Harmonic vertical viscosity & $U_{0} d z / 200$ \\
$\lambda_{\bar{T}}$ & Linear drag coefficient & $0.5 \sigma_{\max }$ \\
& Inverse restoring time & $2 \sigma_{\max }$ \\
& scale & \\
\hline
\end{tabular}

zonal deviations. In this sense the method is similar to the spectral nudging of Thompson et al. (2006). The restoring is diabatic and yields diapycnal fluxes of buoyancy that are certainly present within the ocean mixed layer (Tandon and Garrett 1996; Treguier et al. 1997), and we consider the zonal restoring as a surrogate for more realistic diabatic processes that would retain a surface buoyancy front in the real ocean.

The instability of the flow yields a conversion of potential energy into eddy kinetic energy. Because of the restoring, there is a permanent source of energy that has to be balanced by dissipation to obtain an equilibrated energy budget. Here, we use a Rayleigh damping of zonal and meridional momentum to extract kinetic energy and to damp the inverse energy cascade at the largest scales. The applied time scale is chosen to be proportional to the maximum growth rate, that is, the drag coefficient is set to $\lambda_{u}=0.5 \sigma_{\max }$. In addition to the linear drag, we use biharmonic horizontal and harmonic vertical friction with no-slip boundary conditions at the sidewalls and free-slip at the bottom (viscosities can be found in Table 1). Temperature is advected by a thirdorder upwind advection scheme. No explicit diffusion is used, except in statically unstable conditions $\left(N^{2}<0\right)$, where an implicit vertical diffusion with diffusivity of $1 \times 10^{-2} \mathrm{~m}^{2} \mathrm{~s}^{-1}$ parameterizes convection. Since we do not expect nonhydrostatic effects to become relevant for the parameter range chosen in this study, we use the hydrostatic version of the MITgcm (tests with the nonhydrostatic version do not yield different results).

Snapshots of the equilibrated flow are shown in Figs. 2a-c for one simulation dominated by ageostrophic 
a)
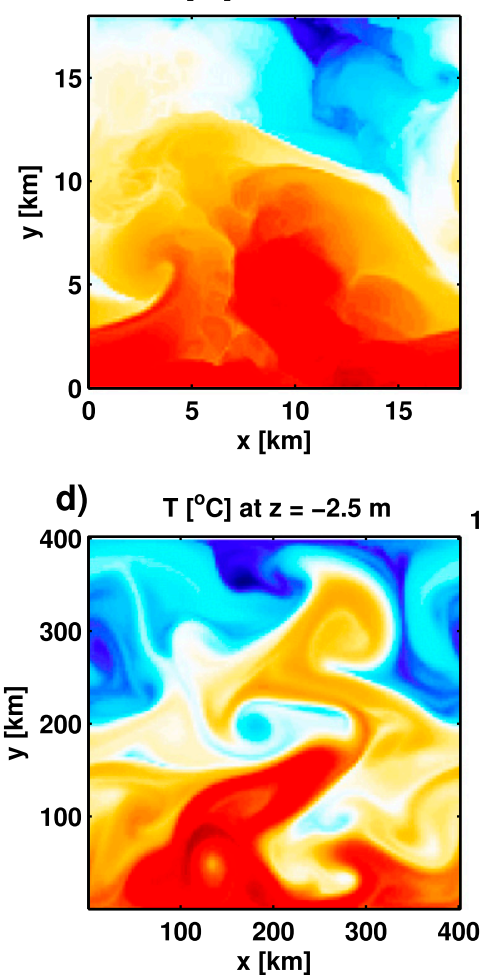

b)

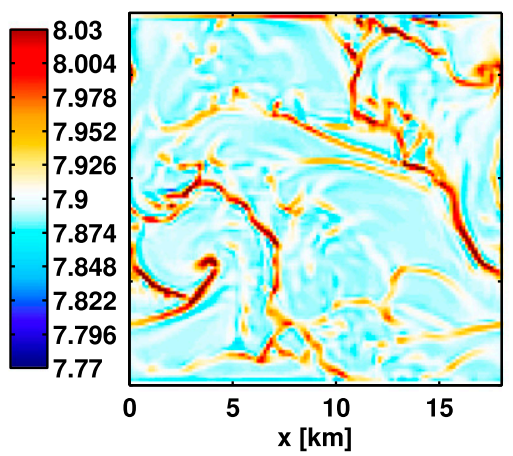

e)

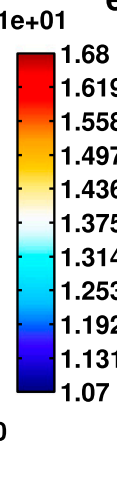

e)

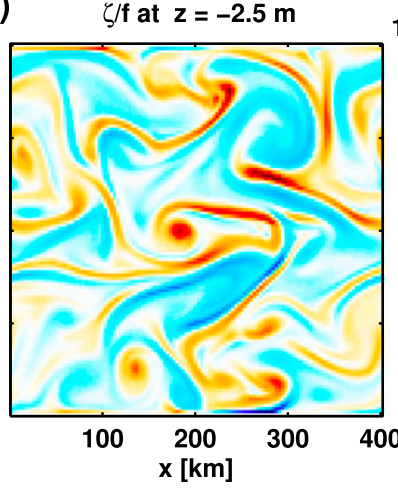

c)
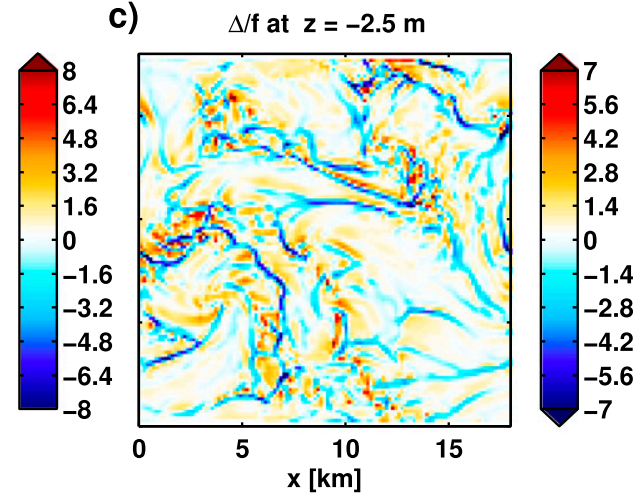

f)

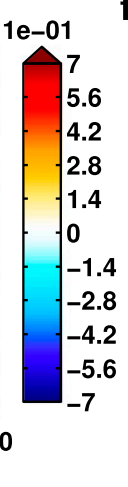

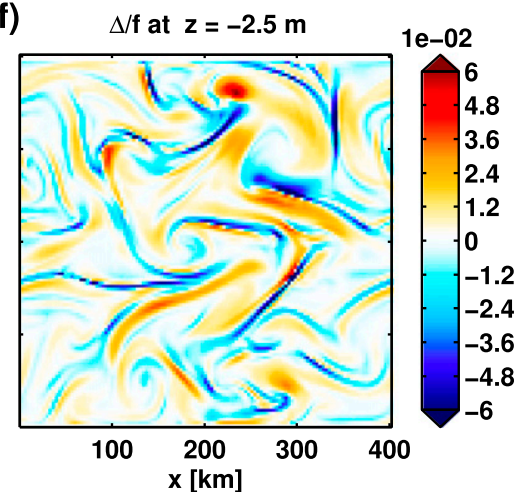

FIG. 2. Instantaneous horizontal sections of (a),(d) temperature, (b),(e) vorticity $\zeta=\partial_{x} v-\partial_{y} u$, and (c),(f) horizontal velocity divergence $\Delta=\partial_{x} u+\partial_{y} v$. The terms $\zeta$ and $\Delta$ are normalized with $f$ and shown at $t=160 \sigma_{\max }^{-1}$ for an experiment with $\mathrm{Ri}_{0}=1$ and $\alpha_{0}=4$ in (a)-(c) and an experiment with $\mathrm{Ri}_{0}=1000$ and $\alpha_{0}=4$ in (d)-(f).

dynamics $\left(\mathrm{Ri}_{0}=1\right.$ and $\left.\alpha_{0}=4\right)$ and in Figs. $2 \mathrm{~d}-\mathrm{f}$ for one with predominantly quasigeostrophic balanced dynamics $\left(\mathrm{Ri}_{0}=1000\right.$ and $\left.\alpha_{0}=0.25\right)$. The snapshots of temperature in Figs. 2a and 2d indicate large eddy activity. Typical for ageostrophic dynamics, the simulation with $\mathrm{Ri}_{0}=1$ and $\alpha_{0}=4$ features a relative vorticity $\zeta=\partial_{x} v-$ $\partial_{y} u$ shown in Fig. $2 \mathrm{~b}$ that is much larger than $f$ within the spiral-like, structured eddies indicating large local Rossby numbers. Within these regions of large relative vorticity, values of the horizontal velocity divergence $\Delta=\partial_{x} u+\partial_{y} v$ are of the same order as $\zeta$ (Fig. 2c). In the simulation with $\mathrm{Ri}_{0}=1000$ and $\alpha_{0}=0.25$, smaller values of $\zeta$ occur, and $\Delta$ is much smaller than the vorticity (Figs. $2 \mathrm{e}, \mathrm{f})$, which is typical for a flow in quasigeostrophic balance.

Figure 3a shows a time series of globally averaged available potential energy (APE), eddy kinetic energy (EKE), mean kinetic energy (MKE), and total energy (TE): TE $=\mathrm{APE}+\mathrm{EKE}+\mathrm{MKE}$ for $\mathrm{Ri}_{0}=1$ and $\alpha_{0}=$ 4. APE is defined as the difference between the globally integrated potential energy $z b$ and a minimum potential energy state. For the latter, we adiabatically rearrange the water parcels such that all horizontal gradients vanish and calculate the global-mean potential energy of this state. Mean kinetic energy is calculated by MKE $=1 / 2\left(\bar{u}^{2}+\bar{v}^{2}\right)$ where $\bar{u}$ and $\bar{v}$ denote zonal averages of the zonal and meridional velocity components $u$ and $v$, respectively. Eddy kinetic energy is determined by $\operatorname{EKE}=1 / 2\left(u^{\prime 2}+v^{\prime 2}\right)$, with $u^{\prime}=\bar{u}-u$ and $v^{\prime}=\bar{v}-v$.

After a period of approximately $20 \sigma_{\max }^{-1}$, the simulation has reached a statistical equilibrium in which the global-mean potential and kinetic energy are fluctuating around their time-mean value without showing a systematic trend. In Fig. 3b, the time series of global-mean Ri and $\alpha$ are shown, both parameters are scaled by their initial value $\mathrm{Ri}_{0}=1$ and $\alpha_{0}=4$. Ri and $\alpha$ are calculated as $\mathrm{Ri}_{0}$ and $\alpha_{0}$ in Eq. (12), but using the globally averaged instead of the initial values of the vertical and meridional stratification $N^{2}$ and $M^{2}$, respectively. While there is hardly any change in $\alpha$, Ri increases by a factor of 7 after baroclinic instability sets in. A time average from $t=40 \sigma_{\max }^{-1}$ to $t=160 \sigma_{\max }^{-1}$ (indicated by the vertical black lines in Fig. 3) is applied for each simulation to obtain diagnosed values for $\mathrm{Ri}$ and $\alpha$ that are used to identify the parameter dependency of the eddy fluxes. For the experiment shown in Fig. 3, this yields $\mathrm{Ri}=6.9$ and $\alpha=$ 3.7. Note that the ratio $\mathrm{Ri} / \mathrm{Ri}_{0}$ becomes smaller in experiments with larger Ri (not shown). 

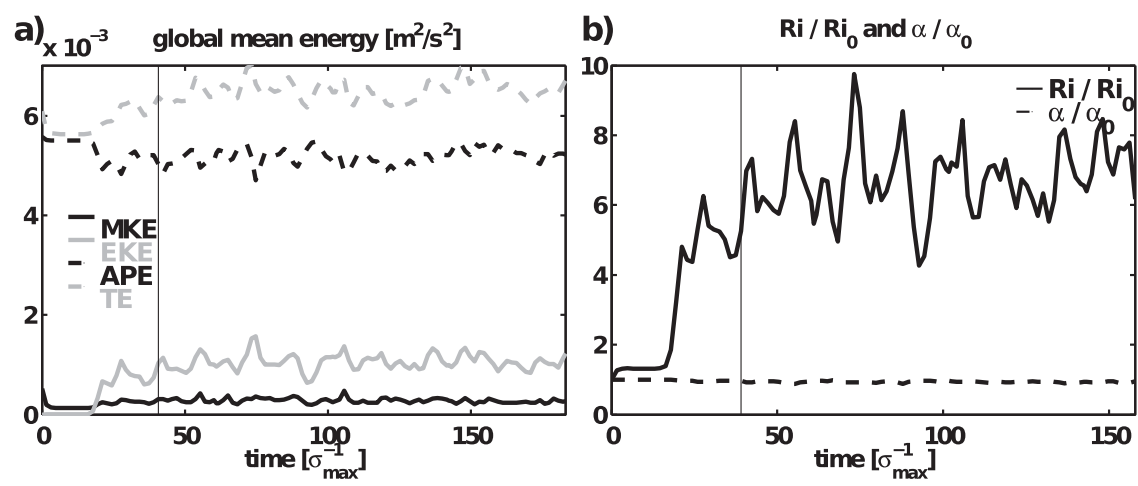

FIG. 3. (a) Global-mean total energy (gray dashed), mean kinetic energy (black solid), eddy kinetic energy (gray solid), and available potential energy (black dashed) as a function of time for an experiment with $\mathrm{Ri}_{0}=1$ and $\alpha_{0}=4$. (b) Diagnosed $\mathrm{Ri}$ (solid) and $\alpha$ (dashed) normalized by their initial values $\mathrm{Ri}_{0}$ and $\alpha_{0}$ as a function of time. Time is scaled by the initial growth rate $\alpha_{\max }$. Vertical black lines indicate the time point from which time averages are applied (see text).

\section{b. Evaluating the parameterizations}

To investigate the dependency of the eddy fluxes on $\mathrm{Ri}$ and $\alpha$, we perform different experiments by varying the initial and restoring temperature $T_{0}$. All other parameters are chosen as detailed in Table 1. Each experiment is integrated over a time period of more than $150 \sigma_{\max }^{-1}$. Time-mean values of $\mathrm{Ri}$ and $\alpha$ are derived as described in the previous section where we use global and time averages of $M^{2}$ and $N^{2}$. This time average is performed over a period of more than $100 \sigma_{\max }^{-1}$ as indicated in Fig. 3 by the vertical black lines and the meridional mean is limited to regions with $y>0.1 L_{y}$ and $y<0.9 L_{y}$, where $L_{y}$ denotes the basin width in order to exclude boundary effects. Eddy fluxes are determined by considering a zonal and time mean of $v, w$, and $b$ and the corresponding deviations to determine $\overline{v^{\prime} b^{\prime}}$ and $\overline{w^{\prime} b^{\prime}}$. An eddy streamfunction $\Psi$ and a diapycnal diffusivity $K_{\text {dia }}$ are calculated from the diagnosed $\overline{v^{\prime} b^{\prime}}$ and $\overline{w^{\prime} b^{\prime}}$ as

$$
\Psi=\frac{\overline{v^{\prime} b^{\prime}} \partial_{z} \bar{b}-\overline{w^{\prime} b^{\prime}} \partial_{y} \bar{b}}{|\nabla \bar{b}|^{2}}, \quad K_{\mathrm{dia}}=-\frac{\overline{v^{\prime} b^{\prime}} \partial_{y} \bar{b}+\overline{w^{\prime} b^{\prime}} \partial_{z} \bar{b}}{|\nabla \bar{b}|^{2}} .
$$

To compare the diagnosed $\overline{v^{\prime} b^{\prime}}, \overline{w^{\prime} b^{\prime}}, \Psi$, and $K_{\mathrm{dia}}$ with the vertical profiles of the parameterizations, we take an additional meridional average of the respective quantities where we again exclude the meridional boundaries as mentioned above.

Note that in their parameterization Fox-Kemper et al. (2008) use a different representation of the streamfunction following Held and Schneider (1999). In this case, the streamfunction $\Psi^{\mathrm{HS}}=-\overline{w^{\prime} b^{\prime}} / \partial_{y} \bar{b}$ is defined by the vertical eddy flux only, and the residual flux is in the horizontal direction [and not in the isopycnal direction as it is if Eq. (13) is used]. Since Fox-Kemper et al. (2008) represent the eddy fluxes by $\Psi^{\mathrm{HS}}$ only, their parameterization is adiabatic. We stress that this is different from our approach. It is important to note that the parameterization based on Eq. (9) referred to as PER as well as the parameterization based on Eq. (6) referred to as ALS are not adiabatic and thus $K_{\text {dia }} \neq 0$.

The parameterizations for $\overline{v^{\prime} b^{\prime}}$ and $\overline{w^{\prime} b^{\prime}}$ based on the analytic solution of the ALS by Stone (1972a) and that based on the PER are given by Eq. (6) and Eq. (9), respectively. In addition, we parameterize the eddy fluxes by a third method very similar to ALS, namely, a numerical solution of the linearized eigenvalue problem (NLS) for a given background state instead of using the approximate solution by Stone (1972a). In NLS, $M^{2}$ and $N^{2}$ are allowed to vary vertically. Details on the method can be found in Thomsen et al. (2014). As before for ALS, we scale the eigenvectors by assuming $v^{\prime}=C_{N} \sigma_{\max } / k_{\max }$, where $\sigma_{\max }$ and $k_{\max }$ are now determined numerically, and $C_{N}$ is a tuning factor. Since the computational costs of this method are very high, it is probably not appropriate to be implemented in a numerical ocean model. Nevertheless, a consideration of NLS enables us to differentiate if differences between diagnosed eddy fluxes and ALS are due to approximations made in Stone (1972a) and how much improvement could be achieved with more accurate eigenfunctions.

We determine $C_{S}$ and $C_{F}$ by a least squares fit between the mean diagnosed and parameterized eddy fluxes. Since we are aiming to achieve a variation of the eddy fluxes over several orders of magnitude, we apply a logarithmic weighting and therefore minimize $\sum_{i}\left[\log _{10}\left(y_{i}\right)-\log _{10}\left(x_{i}\right)-\log _{10}(C)\right]^{2}$ to obtain the fitting constant $C=C_{S}$ or $C=C_{F}$, where $y_{i}$ denotes the 
a)

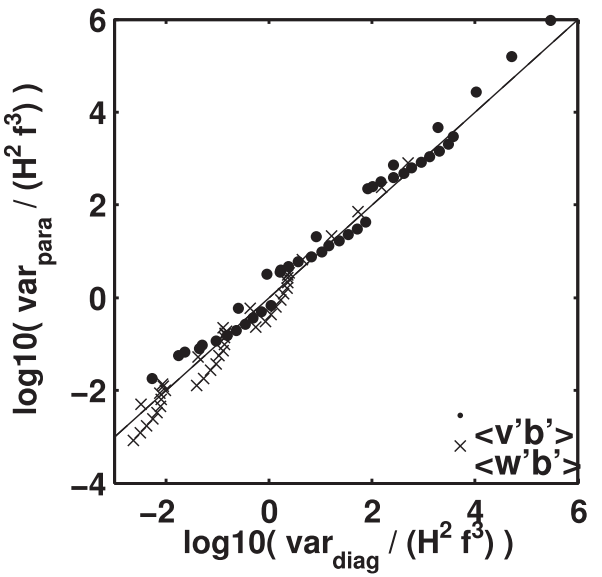

b)

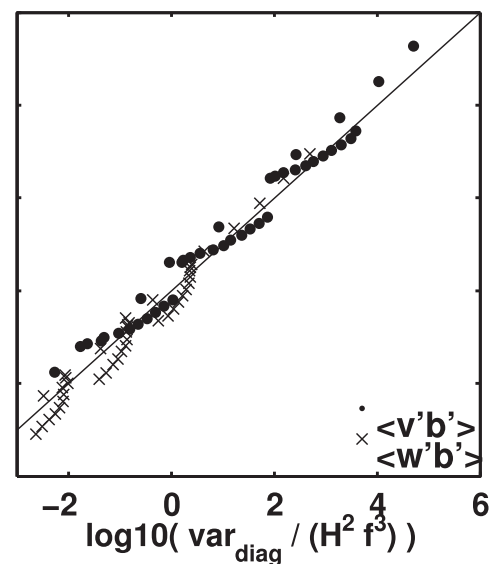

c)

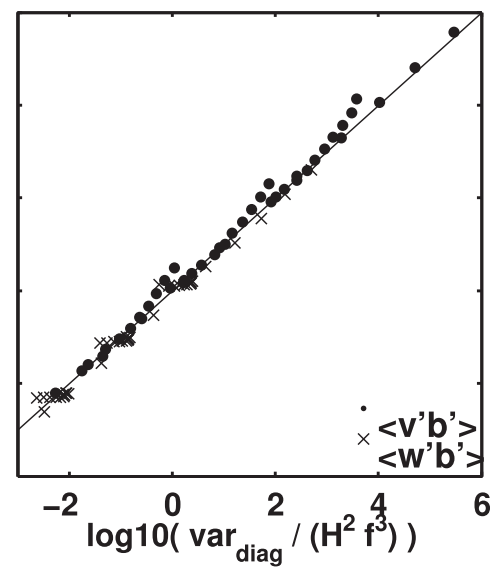

FIG. 4. (a)-(c) Fitting of the different parameterizations for $\overline{v^{\prime} b^{\prime}}$ (dots) and $\overline{w^{\prime} b^{\prime}}$ (crosses). Tuning parameters are obtained by a least squares fit as $C_{S}=0.90$ in (b) and $C_{F}=0.08$ in (c).

diagnosed mean of $\overline{v^{\prime} b^{\prime}}$ and $\overline{w^{\prime} b^{\prime}}$ and $x_{i}$ denotes the parameterized counterpart of ALS or PER, respectively, for an experiment $i$ characterized by a certain $\mathrm{Ri}_{0}$ and $\alpha_{0}$. With this, we obtain $C_{S}=1.1$ and $C_{F}=0.16$ for the corresponding parameterizations. Fox-Kemper et al. (2008) find $C_{F}=0.06$ for their spindown simulations, thus a significantly smaller value.

Note that Fox-Kemper et al. (2008) determine $C_{F}$ by considering $\Psi^{\mathrm{HS}}=-\overline{w^{\prime} b^{\prime}} / \partial_{y} \bar{b}$ instead of considering both $\overline{v^{\prime} b^{\prime}}$ and $\overline{w^{\prime} b^{\prime}}$ as we do here. In the case that $C_{F}$ is determined with $\Psi^{\mathrm{HS}}$ alone as done by Fox-Kemper et al. (2008), we obtain $C_{F}=0.2$. Capet et al. (2008) find values in the range of $0.15-0.25$ and thus 2 to 3 times larger than the value estimated by Fox-Kemper et al. (2008) and within the range that we find for the equilibrated scenario. Similarly, Bachman and Fox-Kemper (2013) find $C_{F}=0.17$ in good agreement with our finding. However, the exact values of $C_{S}$ and $C_{F}$ might strongly depend on the strength of the diabatic effects as can be inferred from sensitivity simulations discussed in the following section. Note that we also find a substantially smaller value in the spindown scenario (see section 4 ) where the diabatic processes are substantially smaller.

In Fig. 4, we show the scaled parameterizations for $\overline{v^{\prime} b^{\prime}}$ and $\overline{w^{\prime} b^{\prime}}$ for the three parameterizations as a function of the diagnosed $\overline{v^{\prime} b^{\prime}}$ and $\overline{w^{\prime} b^{\prime}}$. The closer the points are to the black diagonal line, the better the diagnosed eddy fluxes match their parameterized counterparts. Note that different tuning coefficients would mean a shift parallel to the vertical axis of the points in Fig. 4.

In principle, the quality of the single parameterizations might be inferred from the scatter of the points in Fig. 4, but for a more detailed analysis we want to consider the $\mathrm{Ri}$ and $\alpha$ dependency separately. Plotting the mean values of the profiles of $\overline{v^{\prime} b^{\prime}}, \overline{w^{\prime} b^{\prime}}, \Psi$, and $K_{\text {dia }}$ against Ri in Figs. 5a-d for experiments with $\alpha_{0}=4$ shows the dependency of the eddy fluxes on Ri. A linear regression in these double logarithmic plots yields the exponents $\kappa$ of the leading-order $\mathrm{Ri}^{\kappa}$ dependency of the eddy fluxes. Similarly, we obtain the leading-order $\alpha^{\lambda}$ dependency from a set of experiments with $\mathrm{Ri}_{0}=1$ by a linear regression of the eddy fluxes against $\alpha$ in the double logarithmic plots shown in Figs. 5e-h. It turns out that there is no qualitative change of the Ri dependency for experiments with $\alpha_{0}=1$ and $\alpha_{0}=0.25$, and vice versa there is no change of the $\alpha$ dependency for different $\mathrm{Ri}$ (not shown).

The slopes determined by the linear regressions for Fig. 5 that indicate the leading-order dependency on $\mathrm{Ri}$ and $\alpha$ are given in Table 2. Note that in some cases, the slopes for ALS and PER do not perfectly agree with Eqs. (6) and (9), respectively. The reason for that is that $\alpha$ does not stay strictly constant throughout the experiments shown in Figs. 5a-d and also Ri slightly varies within the experiments for different $\alpha$ shown in Figs. 5e-h. Thus, the eddy fluxes do not only vary due to a change of Ri in Figs. 5a-d, but also due to slight changes of $\alpha$, causing the slopes of ALS and PER to differ from what would be expected for $\alpha=$ const. The same is true for the slopes determined by the experiments shown in Figs. 5e-h. Equation (6) suggests for ALS and large Ri a slope of $\kappa=0.5$ and $\lambda=3$ for $\overline{v^{\prime} b^{\prime}}$ and $\kappa=-0.5$ and $\lambda=2$ for $\overline{w^{\prime} b^{\prime}}$, while Eq. (9) suggests for PER $\kappa=1$ and $\lambda=3$ for $\overline{v^{\prime} b^{\prime}}$ and $\kappa=0$ and $\lambda=2$ for $\overline{w^{\prime} b^{\prime}}$. However, as can be inferred by Table 2, the resulting deviations from the determined slopes to these theoretical slopes for ALS and PER are rather small. 

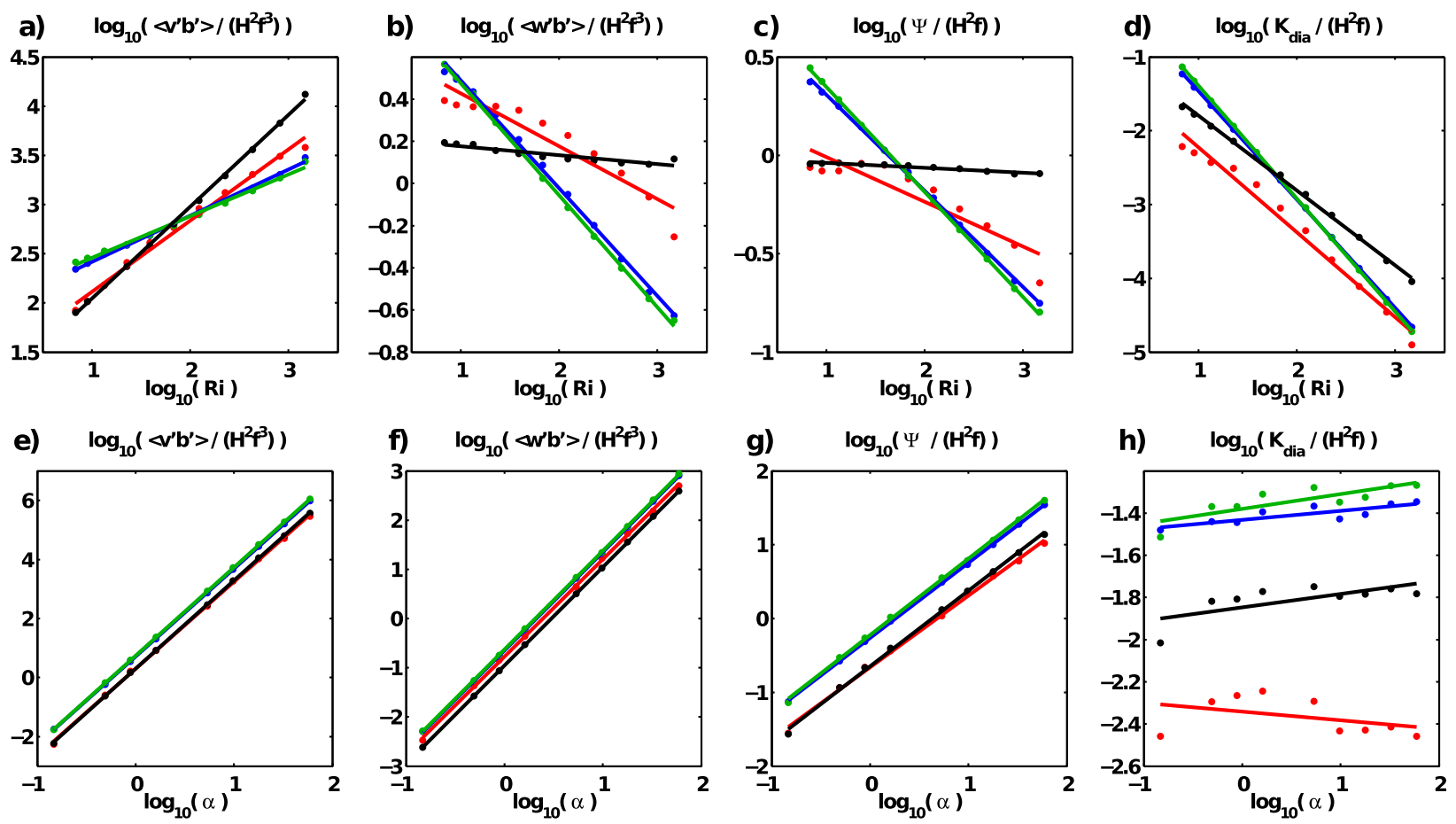

FIG. 5. (a),(e) Meridional and (b),(f) vertical eddy fluxes $\overline{v^{\prime} b^{\prime}}$ and $\overline{w^{\prime} b^{\prime}}$, (c),(g) eddy streamfunction $\Psi$, and (d),(h) diapycnal diffusivity $K_{\text {dia }}$ are plotted in (a)-(d) against Ri for simulations with $\alpha_{0}=4$ and in (e)-(h) against $\alpha$ for simulations with $\mathrm{Ri}_{0}=1$. Red dots indicate diagnosed results, and black, blue, and green dots are the corresponding PER, NLS, and ALS parameterizations. Colored lines denote respective linear least squares fits. The slopes of these fits in the double logarithmic plots indicate the leading-order dependency on $\mathrm{Ri}$ in (a)-(d) and the dependency on $\alpha$ in (e)-(h) (slopes are given in Table 2).

Despite these issues, it can be noted that the Ri dependency of the diagnosed eddy fluxes $\overline{v^{\prime} b^{\prime}}$ and $\overline{w^{\prime} b^{\prime}}$ is not perfectly matched by any parameterization and that the estimated exponents $\kappa$ for the diagnosed eddy fluxes are in between the PER and ALS parameterizations. Furthermore, the numerical simulations indicate a strong decay of $\overline{w^{\prime} b^{\prime}}$ for larger $\mathrm{Ri}$ that is in contradiction to PER. Although the tendency for decreasing $\overline{w^{\prime} b^{\prime}}$ with larger Ri is correctly described by the NLS and ALS, the decrease in the numerical model is not as strong as suggested by these parameterizations. For the eddy streamfunction $\Psi$ and the diapycnal diffusivity $K_{\text {dia }}$, we obtain similar findings, since $\Psi$ and $K_{\text {dia }}$ are functions of $\overline{v^{\prime} b^{\prime}}$ and $\overline{w^{\prime} b^{\prime}}$. However, it should be noted that all parameterizations have a low bias in predicting diffusivities.

Figure 6 shows the vertical structure of the eddy fluxes for $\alpha_{0}=4$ and different Ri. Because there are large variations of the magnitude of the eddy fluxes between the parameterizations, Fig. 6 shows all profiles normalized by their maximum values. The diagnosed profiles resemble what we expect from linear stability analysis. While $\overline{v^{\prime} b^{\prime}}$ is almost constant in the vertical, $\overline{w^{\prime} b^{\prime}}$ has a parabolic vertical dependency with a maximum at middepth (red lines in Figs. 6a,b). The structure of $\overline{v^{\prime} b^{\prime}}$ is quite well matched by ALS, but does not share the same vertical dependency as predicted by PER. All parameterizations capture quite well the diagnosed profile of $\overline{w^{\prime} b^{\prime}}$. Note that we use global averages of $M^{2}$ and $N^{2}$ for PER and ALS. In contrast, we use horizontally averaged profiles of $M^{2}$ and $N^{2}$ in NLS.

The profiles of $\Psi$ and $K_{\text {dia }}$ depend not only on $\overline{v^{\prime} b^{\prime}}$ and $\overline{w^{\prime} b^{\prime}}$, but also on the vertical structure of $M^{2}$ and $N^{2}$. While $M^{2}$ has nearly no vertical structure, $N^{2}$ increases at the top and the bottom (not shown) and therefore influences the structure of $\Psi$ and $K_{\text {dia }}$. The resulting profiles are shown in Figs. 6c and 6d. The vertical structure of $\Psi$ (red line) is quite well captured by NLS and ALS (blue and green lines) in contrast; the profile of the PER parameterization decays too strong at the top and at the bottom.

TABLE 2. Exponents $\kappa$ and $\lambda$ of the estimated $\mathrm{Ri}^{\kappa}$ and $\alpha^{\lambda}$ dependency of the eddy fluxes, streamfunction, and diapycnal diffusivity in the forced dissipative scenario.

\begin{tabular}{lrrrrcccc}
\hline & $\kappa_{\text {diag }}$ & $\kappa_{\text {PER }}$ & $\kappa_{\mathrm{NLS}}$ & $\kappa_{\mathrm{ALS}}$ & $\lambda_{\text {diag }}$ & $\lambda_{\text {PER }}$ & $\lambda_{\mathrm{NLS}}$ & $\lambda_{\mathrm{ALS}}$ \\
$\overline{\overline{v^{\prime} b^{\prime}}}$ & 0.7 & 0.9 & 0.5 & 0.4 & 3.0 & 3.0 & 3.0 & 3.0 \\
$\overline{w^{\prime} b^{\prime}}$ & -0.3 & -0.0 & -0.5 & -0.5 & 2.0 & 2.0 & 2.0 & 2.0 \\
$\Psi$ & -0.2 & -0.0 & -0.5 & -0.5 & 1.0 & 1.0 & 1.0 & 1.0 \\
$K_{\text {dia }}$ & -1.2 & -1.0 & -1.5 & -1.5 & 0.0 & 0.1 & 0.0 & 0.1 \\
\hline
\end{tabular}



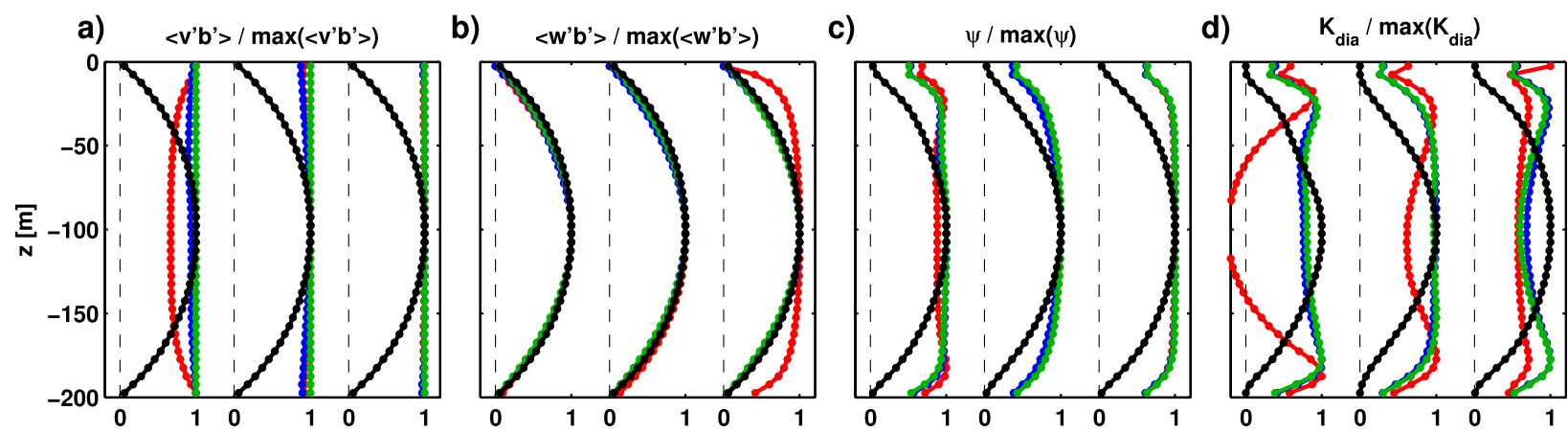

FIG. 6. Vertical profiles for (a) $\overline{v^{\prime} b^{\prime}}$, (b) $\overline{w^{\prime} b^{\prime}}$, (c) $\Psi$, and (d) $K_{\text {dia }}$ for the diagnosed eddy fluxes (red) and the PER, NLS, and ALS parameterizations (black, blue, and green). All profiles are normalized by their maximum value and shifted for each experiment, while the dashed black lines indicate the respective zero lines. The three experiments are for $\alpha_{0}=4$, and for $\mathrm{Ri}$ varying from left to right as $\mathrm{Ri}=3.2$, $\mathrm{Ri}=46$, and $\mathrm{Ri}=1200$

While the structure of $K_{\mathrm{dia}}$ is not matched at all by PER, it is also only partly matched by ALS and NLS. At top and bottom ALS and NLS closely follow the diagnosed profile, but at middepth they underestimate the minimum of $K_{\text {dia. }}$. Note that $K_{\text {dia }}$ becomes negative for small $\mathrm{Ri}$ that is probably due to the influence of rotational eddy fluxes (Eden and Greatbatch 2008a). It might also be that the strong middepth minimum would be less marked if the rotational eddy fluxes are subtracted from the total eddy fluxes, such that the profiles of NLS and ALS match better the diagnosed profiles of $K_{\text {dia }}$, but we have made no attempt to do so.

\section{c. Sensitivity simulations in the forced dissipative scenario}

It is assumed in ALS and PER that the functional relationship between the eddy fluxes and $\mathrm{Ri}$ and $\alpha$ does not depend on parameters controlling dissipation, diffusion, or forcing. To evaluate this hypothesis, we perform different sensitivity studies where we vary the linear drag, zonal restoring, resolution, and small-scale dissipation. How these sensitivity experiments vary with respect to the standard configuration (STD) discussed so far is depicted in Table 3. The magnitude of the eddy fluxes of these experiments is shown in Fig. 7, and the estimated Ri dependency is depicted in Table 4.

Since the energy conversion between potential and kinetic energy is influenced by the temperature restoring and the linear damping, we test the sensitivity of the $\mathrm{Ri}$ dependency of the eddy fluxes for different restoring time scales $\lambda_{\bar{T}}$ and drag coefficients $\lambda_{u}$ (experiments ZR and LD, respectively). These experiments indicate that $\lambda_{\bar{T}}$ has a minor influence on the Ri dependency and the magnitude of the eddy fluxes. In contrast, we find that $\lambda_{u}$ has an influence on the magnitude of the eddy fluxes. This influence is strongest for large $\mathrm{Ri}$. Therefore, $\lambda_{u}$ changes both the Ri dependency and the mean magnitude of the eddy fluxes. As a consequence, the proportionality constants $C_{S}$ and $C_{F}$ decrease for increasing $\lambda_{u}$. The three simulations LD25, STD, and LD75 suggest that $C_{S}$ and $C_{F}$ are inversely proportional to $\lambda_{u}$. Note that not only the eddy fluxes but also the EKE decreases with increasing $\lambda_{u}$ (not shown), and $\lambda_{u}$ has therefore a strong influence on the entire eddy activity.

TABLE 3. Parameters for different sensitivity experiments in the forced dissipative scenario. Note that $k_{\max }$ and $\sigma_{\max }$ are derived by Eq. (5) using the initial $\mathrm{Ri}_{0}$ and $\alpha_{0}$ and $U_{0}=\alpha_{0} H f$ with $\alpha_{0}=4$ for all listed experiments.

\begin{tabular}{|c|c|c|c|c|c|c|c|}
\hline Name & $\left(n_{x}, n_{y}, n_{z}\right)$ & $\lambda_{\bar{T}} / \sigma_{\max }$ & $\lambda_{u} / \sigma_{\max }$ & $\lambda_{\bar{u}} / \sigma_{\max }$ & $k_{\max } d x$ & $U_{0} d x^{3} / A_{4}$ & $U_{0} d z / A_{v}$ \\
\hline STD & $(120,120,40)$ & 2 & 0.5 & 0 & 0.21 & 20 & 200 \\
\hline LD25 & $(120,120,40)$ & 2 & 0.25 & 0 & 0.21 & 20 & 200 \\
\hline LD75 & $(120,120,40)$ & 2 & 0.75 & 0 & 0.21 & 20 & 200 \\
\hline ZR15 & $(120,120,40)$ & 1.5 & 0.5 & 0 & 0.21 & 20 & 200 \\
\hline ZR25 & $(120,120,40)$ & 2.5 & 0.5 & 0 & 0.21 & 20 & 200 \\
\hline $\mathrm{H} 2$ & $(240,240,40)$ & 2 & 0.5 & 0 & 0.10 & 20 & 200 \\
\hline $\mathrm{H} 4 \mathrm{~V} 2$ & $(512,512,80)$ & 2 & 0.5 & 0 & 0.05 & 1 & 10 \\
\hline CW2 & $(120,120,40)$ & 2 & 0.5 & 0 & 0.42 & 20 & 200 \\
\hline $\mathrm{ZD}$ & $(120,120,40)$ & 2 & 0 & 2 & 0.42 & 2 & 200 \\
\hline $\mathrm{ZDH} 2$ & $(240,240,40)$ & 2 & 0 & 2 & 0.42 & 2 & 200 \\
\hline ZDV2 & $(120,120,80)$ & 2 & 0 & 2 & 0.42 & 2 & 200 \\
\hline
\end{tabular}


a) $\log _{10}\left(<v^{\prime} b^{\prime}>/\left(H^{2} f^{3} \alpha^{3}\right)\right)$

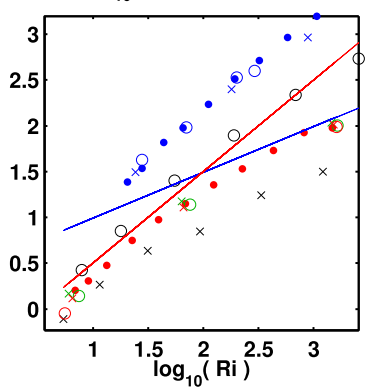

b) $\log _{10}\left(<w^{\prime} b^{\prime}>/\left(H^{2} f^{3} \alpha^{2}\right)\right)$

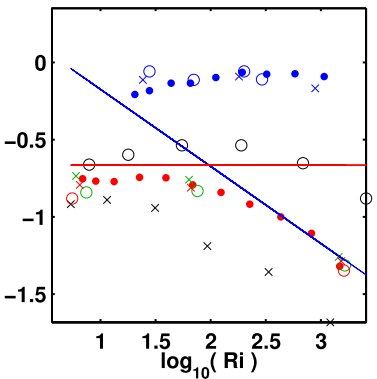

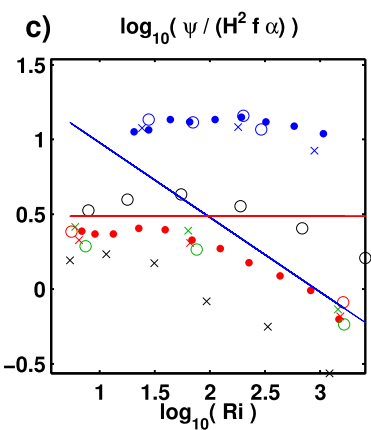

d) $\quad \log _{10}\left(K_{\text {dia }} /\left(H^{2} \mathrm{f}\right)\right)$

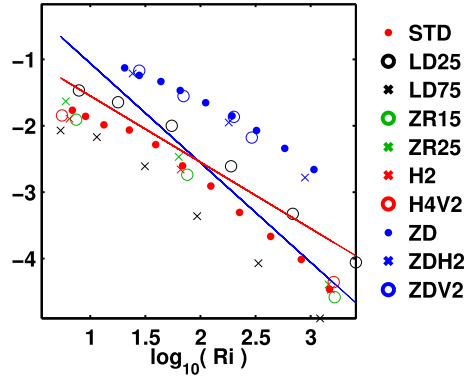

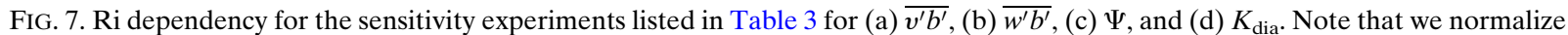
the eddy fluxes not only by $H$ and $f$, but also by the nondimensional parameter $\alpha$ in accordance to the respective dependency of the eddy fluxes on $\alpha$. The marker associated with the different experiments is depicted in the legend. Solid red lines indicate the slopes with respect to PER and solid blue lines with respect to ALS.

We furthermore test the sensitivity with respect to increased resolution and reduced horizontal and vertical friction by reducing the viscosities and applying free-slip boundary conditions. We find small sensitivities of the eddy fluxes for a doubled horizontal resolution (H2) and a fourth time increased horizontal and a doubled vertical resolution (H4V2) to STD.

Instead of using the local velocity values for the Rayleigh damping, we use the zonal-mean velocity as damping velocity in different sets of experiments (ZD, $\mathrm{ZDH} 2$, and ZDV2) with a proportionality constant $\lambda_{\bar{u}}=2 \sigma_{\max }$. This type of damping is similar to the zonalmean restoring of temperature used in all simulations of the forced dissipative scenario. Note that it extracts energy predominantly at large scales. The damping at intermediate and small scales is reduced by this zonally averaged drag in comparison to the classical Rayleigh damping used in STD. While these simulations roughly reflect the $\mathrm{Ri}$ dependency of the experiments with the standard zonal drag, the magnitude of the eddy fluxes is roughly a factor of 3 larger for the experiments with the zonally averaged drag. Doubling the horizontal resolution (ZDH2) or the vertical resolution (ZDV2) does not show much difference with respect to the standard resolution (ZD). We also perform sensitivity simulations with different meridional density gradients $\left(\alpha_{0}=0.25\right.$ and $\alpha_{0}=1$ ) in the STD configuration (not shown), but we do not find any qualitative sensitivities of the $\mathrm{Ri}$ dependency for the experiments with different $\alpha_{0}$.

With respect to the quality of the different parameterizations, we note that the Ri dependency of PER better matches than ALS for the experiments with reduced linear drag and for the simulations with the zonally averaged drag (see Fig. 7 and Table 4). While the largest deviations between the eddy fluxes and PER occur for large $\mathrm{Ri}$, these differences nearly vanish for the experiments with the reduced linear drag. The ALS parameterization best matches for experiments with large linear drag coefficients and at large $\mathrm{Ri}$.

Note that there are hardly any changes in the vertical structure functions for $\overline{v^{\prime} b^{\prime}}$ and $\overline{w^{\prime} b^{\prime}}$ between the different sensitivity experiments (not shown). Especially, we find a nearly constant vertical profile for $\overline{v^{\prime} b^{\prime}}$ throughout all simulations except for those with the smallest Ri. As shown in Fig. $6, \overline{v^{\prime} b^{\prime}}$ is slightly smaller at middepth for small Ri. We find this shape of $\overline{v^{\prime} b^{\prime}}$ for $\mathrm{Ri}_{0}=1$ in all sensitivity simulations. Therefore, $\overline{v^{\prime} b^{\prime}}$ is still curved in the opposite direction than PER suggests for small Ri. Besides these small deviations from the constant profile for small $\mathrm{Ri}$, the vertically constant profile of ALS quite well reproduces the diagnosed profiles.

\section{Baroclinic instabilities in a spindown scenario}

\section{a. Numerical simulations}

To simulate mixed layer instabilities at a restratifying density front, we choose a setup similar to that used in

TABLE 4. Estimated functional relationship of $\overline{v^{\prime} b^{\prime}}$ and $\overline{w^{\prime} b^{\prime}}$ on Ri and proportionality constants $C_{S}$ and $C_{F}$ estimated for the respective sensitivity experiments listed in Table 3 and shown in Fig. 7. Since we normalize here the eddy fluxes by the respective $\alpha$ dependency, the exponents that indicate the Ri dependency for STD slightly differ from those that can be found in Table 2 .

\begin{tabular}{lcccc}
\hline \hline Name & $\overline{v^{\prime} b^{\prime}} /\left(H^{2} f^{3} \alpha^{3}\right)$ & $\overline{w^{\prime} b^{\prime}} /\left(H^{2} f^{3} \alpha^{2}\right)$ & $C_{S}$ & $C_{F}$ \\
\hline STD & $0.43 \mathrm{Ri}^{0.79}$ & $0.32 \mathrm{Ri}^{-0.21}$ & 1.3 & 0.15 \\
LD25 & $0.51 \mathrm{Ri}^{0.92}$ & $0.33 \mathrm{Ri}^{-0.08}$ & 3.0 & 0.29 \\
LD75 & $0.34 \mathrm{Ri}^{0.66}$ & $0.28 \mathrm{Ri}^{-0.34}$ & 0.6 & 0.08 \\
ZR15 & $0.34 \mathrm{Ri}^{0.79}$ & $0.27 \mathrm{Ri}^{-0.21}$ & 1.2 & 0.12 \\
ZR25 & $0.44 \mathrm{Ri}^{0.77}$ & $0.33 \mathrm{Ri}^{-0.23}$ & 1.3 & 0.15 \\
H2 & $0.35 \mathrm{Ri}^{0.79}$ & $0.29 \mathrm{Ri}^{-0.22}$ & 1.2 & 0.13 \\
H4V2 & $0.22 \mathrm{Ri}^{0.83}$ & $0.18 \mathrm{Ri}^{-0.19}$ & 0.9 & 0.09 \\
ZD & $1.10 \mathrm{Ri}^{1.06}$ & $0.54 \mathrm{Ri}^{0.07}$ & 11.5 & 1.07 \\
ZDH2 & $1.63 \mathrm{Ri}^{0.95}$ & $0.89 \mathrm{Ri}^{-0.03}$ & 11.9 & 0.98 \\
ZDV2 & $1.51 \mathrm{Ri}^{1.00}$ & $0.91 \mathrm{Ri}^{-0.02}$ & 11.0 & 1.12 \\
\hline
\end{tabular}


Fox-Kemper et al. (2008) and Bachman and FoxKemper (2013) to infer the magnitude and vertical structure of the eddy fluxes. While Fox-Kemper et al. (2008) directly diagnose the eddy buoyancy fluxes in the spindown scenario, Bachman and Fox-Kemper (2013) use the eddy fluxes of several passive tracers to estimate a mixing tensor common to all passive tracers, which is assumed to be also representative for buoyancy. This allows the authors to infer the eddy buoyancy fluxes from the diagnosed tensor elements. Here, however, we diagnose the eddy buoyancy fluxes directly as done by Fox-Kemper et al. (2008).

As in the setup of section 3, the model domain consists of a reentrant zonal channel with solid walls at meridional boundaries. To prevent effects from the solid meridional boundaries, we limit the zonal jet and thereby the location where the instabilities grow to a region of width $L_{f}$ at the center of the channel. This is done by choosing $b_{0}=N_{0}^{2}(z+H)+\left[\left(L_{f} M_{0}^{2}\right) / 2\right] \tanh \left\{2\left[y-\left(L_{y} / 2\right)\right] / L_{f}\right\}$, where $L_{y}$ denotes the width of the channel in meridional direction. This initial condition deviates slightly from that considered by Eady (1949) and Stone (1966), who assumed $\partial_{y y} b_{0}=0$ and $\partial_{y} b_{0}=M_{0}^{2}$, but we will focus our analysis on the center of the front where these conditions are fulfilled. To dissipate momentum, we use harmonic friction with a viscosity depending on the resolved motion after Smagorinsky (1963) with a "Smagorinsky coefficient" of one that was also used by Fox-Kemper et al. (2008). No-slip conditions are applied at the sidewalls but free slip at the bottom. No other boundary conditions are used for momentum or density. As before, we use temperature as the only active tracer and a linear equation of state. Because of the numerical dissipation of the applied third-order upwind advection scheme, we do not use any explicit diffusion. All simulations for the spindown experiments are performed with the nonhydrostatic version of the MITgcm, although we do not expect nonhydrostatic effects to be relevant for the parameter range considered in this study.

We vary the initial stratification to obtain $\mathrm{Ri}_{0}=1$ to $\mathrm{Ri}_{0}=160$ and $\alpha_{0}=4$ in a first set of experiments. Correspondingly, in a second set of experiments with $\mathrm{Ri}_{0}=1$, we vary $\alpha_{0}$ from $\alpha_{0}=0.2$ to $\alpha_{0}=10$. The horizontal resolution is set to $\Delta x=\pi /\left(5 k_{\max }\right)(128$ points in $x$ and $y)$ and the vertical resolutions to $\Delta z=5 \mathrm{~m}$ (60 levels). The basin depth of $H=300 \mathrm{~m}$ and the Coriolis parameter of $f=7.29 \times 10^{-5} \mathrm{~s}^{-1}$ are not varied.

Small white-noise perturbations of $O\left(1 \times 10^{-3} \mathrm{~K}\right)$ in the initial conditions lead to exponentially growing baroclinic instabilities. In contrast to the simulations in the equilibrated scenario, there is no source of potential energy, and baroclinic instability induces a slumping of the temperature front by converting all APE into EKE.
Figure 8 shows that this restratification process takes place on a time scale of about $10 \sigma_{\max }^{-1}$ that corresponds to a few days for the chosen parameters. As in the equilibrated scenario for $\mathrm{Ri}=O(1)$, the flow features local Rossby numbers $\zeta / f=\left(\partial_{x} v-\partial_{y} u\right) / f>1$ and a normalized horizontal velocity divergence $\Delta / f=\left(\partial_{x} u+\partial_{y} v\right) / f$ of the same order of magnitude. Both indicate ageostrophic dynamics. For larger $\mathrm{Ri}$, and therefore quasigeostrophic conditions, $\zeta / f$ is smaller than one and $\Delta \ll \zeta$ (not shown).

Figure 8 shows that the flow field is dominated by large individual eddies, and the same is true for the magnitude of the eddy fluxes (not shown). Since the occurrence of single eddy events is a random process, it is necessary to average over these events. In the equilibrated scenario, a temporal mean over some eddy time scales is sufficient to exclude single eddy events, but the strong time dependencies in the spindown scenario rules out this possibility here. Instead, we perform 10 ensemble simulations for each experiment characterized by its initial $\mathrm{Ri}_{0}$ and $\alpha_{0}$. Each ensemble member only differs in the small random perturbations added to the initial temperature.

In Fig. 9a, we show an ensemble-averaged time series of the conversion of APE into EKE by baroclinic instability for an experiment with $\mathrm{Ri}_{0}=1$ and $\alpha_{0}=4$. During the restratification phase, the MKE stays constant until boundary effects become important, suggesting that there is no significant interaction between the eddies and the mean current. Total mechanical energy (TE) is dissipated due to the applied viscous damping. Figures $9 \mathrm{~b}$ and $9 \mathrm{c}$ show time series of $\mathrm{Ri}$ and $\alpha$, respectively, averaged over the domain. In accordance with the rather small changes in MKE, $\alpha$, or equivalently the meridional buoyancy gradient, hardly changes. The large increase in $\mathrm{Ri}$ soon after the onset of the restratification is mainly caused by changes in the vertical stratification.

\section{b. Evaluating the parameterizations}

In contrast to a quasi-stationary system as considered in section 3, a time-dependent system requires slight changes in our analysis to determine the diagnosed and parameterized eddy fluxes. Most of the diagnostics described in the following are performed similar as in FoxKemper et al. (2008) to allow for a comparison between their and our results. We use a zonal average to estimate mean quantities. By doing so, we obtain eddy fluxes that are two-dimensional and change in time. We furthermore average $\overline{v^{\prime} b^{\prime}}$ and $\overline{w^{\prime} b^{\prime}}$ over $y$ within the active area of the eddies because we are interested in the mean effect of the eddies. Thus, the meridional averaging is restricted to the center of the front [all points for which 


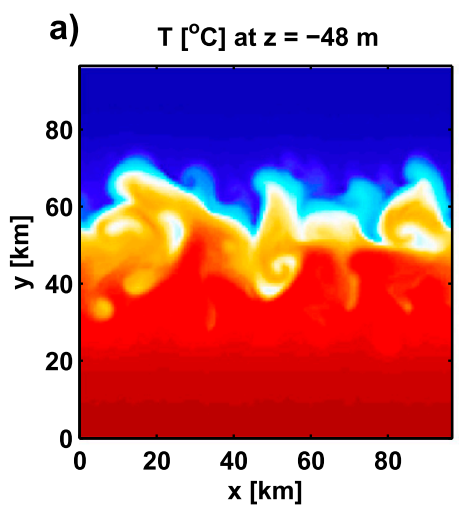

d) $\quad T\left[{ }^{\circ} \mathrm{C}\right]$ at $\mathrm{z}=-48 \mathrm{~m}$

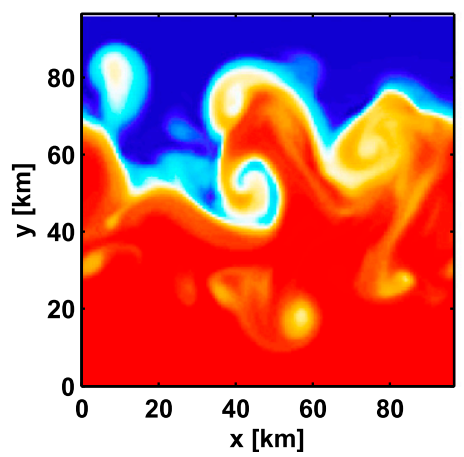

b)

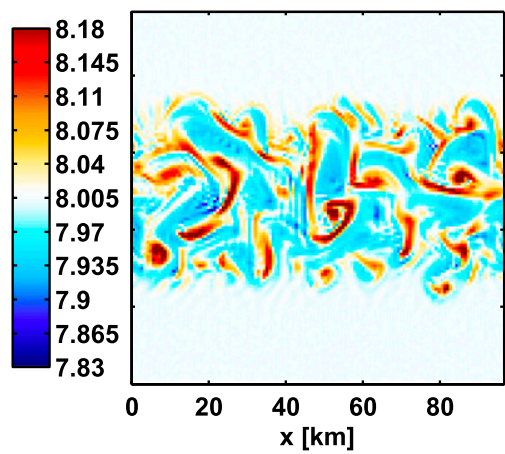

e)

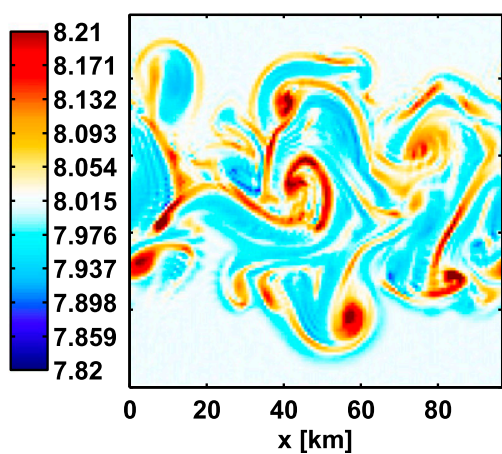

c)

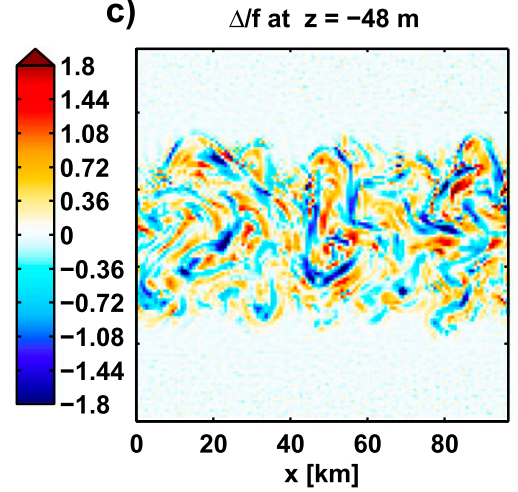

1e-01

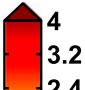

$-3.2$

$-1.6$

$-0.8$

$-\begin{aligned} & 0 \\ & -0.8\end{aligned}$

$-1.6$

$-2.4$

$-3.2$

$-4$

FIG. 8. Instantaneous horizontal sections of (a),(d) temperature, (b),(e) vorticity $\zeta=\partial_{x} v-\partial_{y} u$, and (c),(f) horizontal velocity divergence $\Delta=\partial_{x} u+\partial_{y} v$. The terms $\zeta$ and $\Delta$ are normalized with $f$ and shown for an experiment of the spindown scenario with initial $\mathrm{Ri}_{0}=1$ and $\alpha_{0}=4$. (a)-(c) show a snapshot at the beginning of the averaging period $\left(t=17 \sigma_{\max }^{-1}\right)$ and (d)-(f) show a snapshot at the end of the averaging period $\left(t=28 \sigma_{\text {max }}^{-1}\right)$.

$\left.\overline{M^{2}}<0.1 \max \left(\overline{M^{2}}\right)\right]$. Finally, we perform an average over 10 ensemble simulations as described above.

Such averages over the frontal width of $M^{2}$ and $N^{2}$ are also performed to diagnose $\mathrm{Ri}$ and $\alpha$ given by Eq. (12).
The diagnosed Ri and $\alpha$ enter Eqs. (6) and (9) to determine $\overline{v^{\prime} b^{\prime}}$ and $\overline{w^{\prime} b^{\prime}}$ for ALS and PER, respectively. For NLS, we calculate $\overline{v^{\prime} b^{\prime}}$ and $\overline{w^{\prime} b^{\prime}}$ with the use of the horizontally (over the frontal width) averaged vertical a) global mean energy $\left[\mathrm{m}^{2} / \mathrm{s}^{2}\right]$

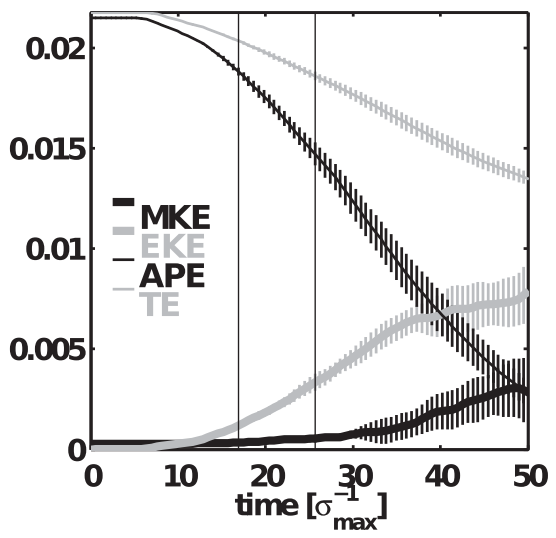

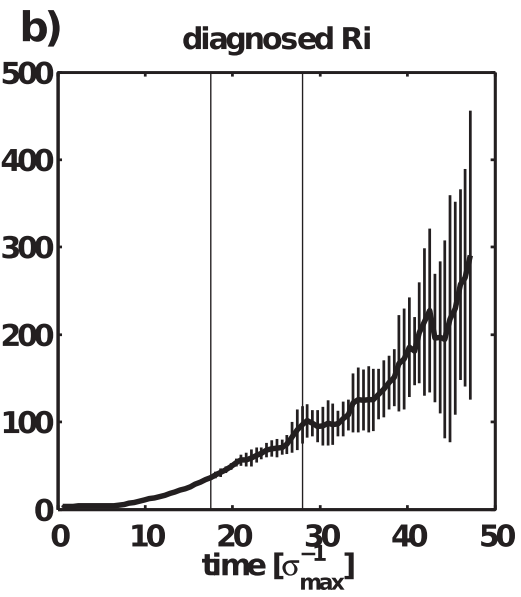

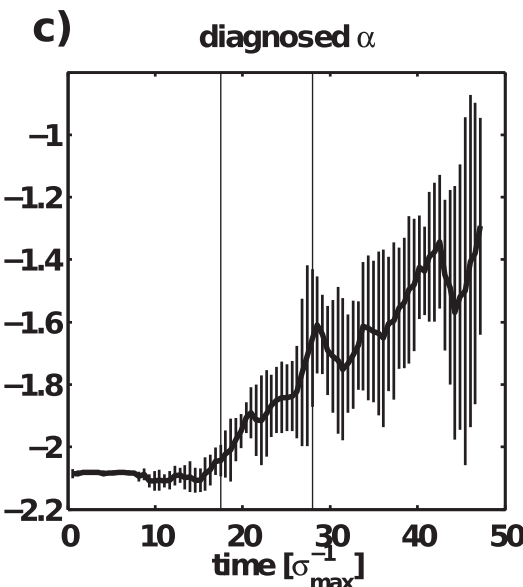

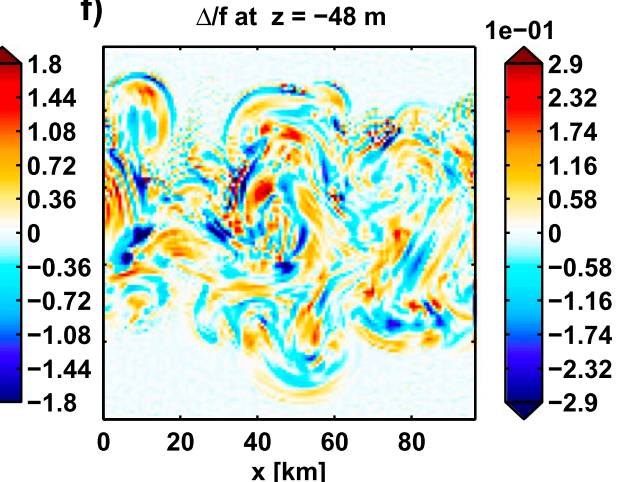

FIG. 9. (a) Time dependency of global-mean total mechanical energy (gray thin line), mean kinetic energy (black thick line), eddy kinetic energy (gray thick line), and available potential energy (black thin line) for an experiment in the spindown scenario with $\mathrm{Ri}_{0}=1$ and $\alpha_{0}=4$. The time dependency of (b) Ri and (c) $\alpha$. Small vertical lines denote the standard deviation of the ensemble spread. Time is scaled by the initial growth rate $\sigma_{\text {max }}$. Vertical black lines indicate the period over which the time average is taken to diagnose the eddy fluxes (see text for details). 

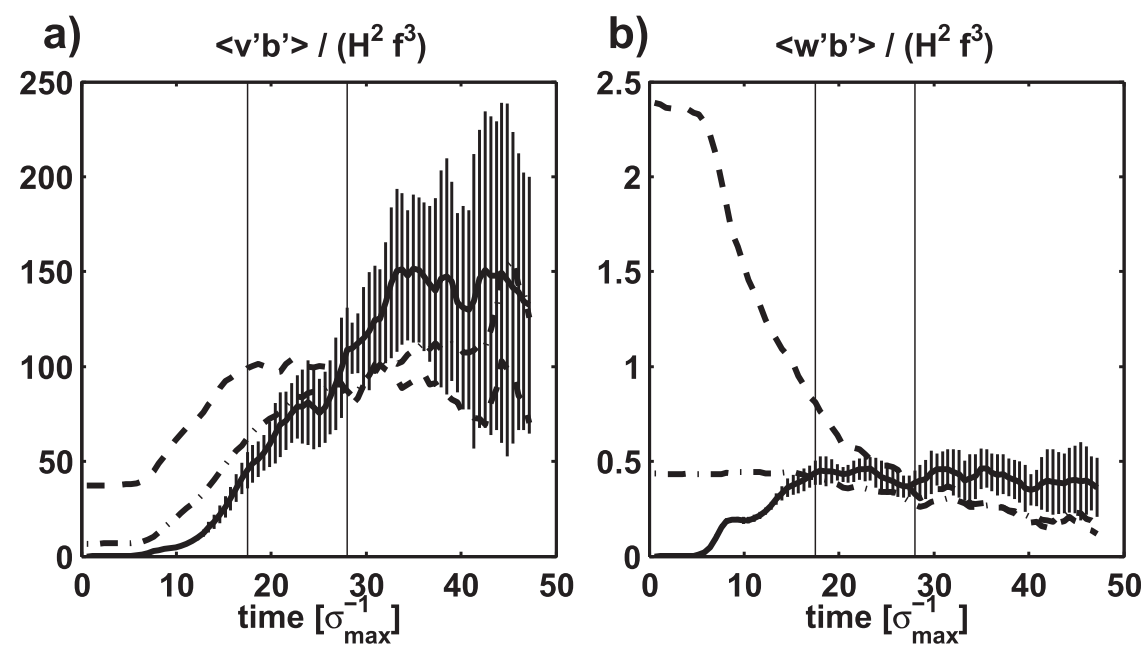

FIG. 10. Vertical maximum of the eddy fluxes (a) $\overline{v^{\prime} b^{\prime}}$ and (b) $\overline{w^{\prime} b^{\prime}}$ for an experiment with $\mathrm{Ri}_{0}=1$ and $\alpha_{0}=4$ as a function of time. Solid lines denote the ensemble-mean and mean over the restratification region (see text for details), while the ensemble means of ALS and PER are denoted by dashed and dashed-dotted lines, respectively. The small vertical lines indicate the standard deviation over 10 ensemble simulations. Time is scaled by the initial growth rate $\sigma_{\max }$ for ALS and PER, respectively. The period of the restratification phase determined as detailed in the text is indicated by vertical black lines.

profiles of $M^{2}$ and $N^{2}$. Finally, we calculate the ensemble mean of the diagnosed and parameterized eddy fluxes to eliminate the effect of single eddy events as described above.

Figure 10 shows the time evolution of the vertical maximum of the eddy fluxes for a specific set of ensemble experiments. In the initial phase, $\overline{v^{\prime} b^{\prime}}$ and $\overline{w^{\prime} b^{\prime}}$ are zero as long as the eddies have not reached finite amplitude yet. After a time of roughly $5 \sigma_{\max }^{-1}, \overline{v^{\prime} b^{\prime}}$ and $\overline{w^{\prime} b^{\prime}}$ start to increase. While $\overline{w^{\prime} b^{\prime}}$ saturates after approximately $15 \sigma_{\max }^{-1}, \overline{v^{\prime} b^{\prime}}$ further increases until $30 \sigma_{\max }^{-1}$.

Fox-Kemper et al. (2008) restrict their analysis on the restratification phase of the eddies. Therefore, they define a time period that starts as soon as the eddies have reached finite amplitude and ends when the eddies reach the meridional boundaries. We also only consider times for which $\sqrt{\overline{v^{2}}}>0.1 U_{0}$ for half of the mixed layer grid points and where $\left[\Delta T(t)-\Delta T\left(t_{0}\right)\right] / \Delta T\left(t_{0}\right)<0.03$ with $\Delta T=\left\langle T\left(y=0.08 L_{y}\right)-T\left(y=0.92 L_{y}\right)\right\rangle^{x z}$, denoting the zonally and depth-averaged temperature difference near the boundaries and $t_{0}$ the initial time. This period is indicated in Figs. 9 and 10 by vertical black lines. In the following, we apply time averages over this period to consider the effect of the eddy restratification.

To scale the eddy fluxes, we proceed similar as in the equilibrated scenario. As before, we minimize $\sum_{i}\left[\log _{10}\left(y_{i}\right)-\log _{10}\left(x_{i}\right)-\log _{10}(C)\right]^{2}$ with respect to the tuning constant $C$ for each parameterization where $y_{i}$ denotes the diagnosed $\overline{v^{\prime} b^{\prime}}$ or $\overline{w^{\prime} b^{\prime}}$ and $x_{i}$ the parameterized counterpart for an experiment $i$. We obtain $C_{S}=0.90$ and $C_{F}=0.08$. Hence, $C_{S}$ is quite similar to the value we obtain for the equilibrated scenario for ALS. In contrast, $C_{F}$ is slightly smaller than the value we find for the equilibrated scenario but still a bit larger than the value of $C_{F}=0.06$ determined by Fox-Kemper et al. (2008). Note that we obtain the same value for $C_{F}$ if we consider $\Psi^{\mathrm{HS}}$ to determine $C_{F}$ instead of considering both $\overline{v^{\prime} b^{\prime}}$ and $\overline{w^{\prime} b^{\prime}}$ as done by Fox-Kemper et al. (2008). Our estimate of $C_{F}$ also agrees well with findings by Bachman and FoxKemper (2013).

Figures 11a-d show the Ri dependency of the eddy fluxes in the spindown scenario. As noted above, $K_{\text {dia }}$ cannot entirely be associated with a diapycnal flux since it additionally represents the effect of the timedependent restratification. In such a scenario, $K_{\text {dia }}$ would also be nonzero if the flow is entirely adiabatic. The ensemble-averaged vertical mean of the eddy flux profiles are shown as a function of Ri for experiments with $\alpha_{0}=4$ and varying $\mathrm{Ri}_{0}$ from $\mathrm{Ri}_{0}=1$ to $\mathrm{Ri}_{0}=160$. Similar to that, Figs. 11e-h show the dependency of the eddy fluxes on $\alpha$ for a second set of experiments with $\mathrm{Ri}_{0}=1$ and $\alpha_{0}$ varying from $\alpha_{0}=0.4$ to $\alpha_{0}=4$. We obtain the exponents $\kappa$ and $\lambda$ of the leading-order $\mathrm{Ri}^{\kappa}$ and $\alpha^{\lambda}$ dependency by a least squares fit and show them in Table 5. Note that as before in the equilibrated scenario, the estimated slopes for ALS and PER slightly differ from what would be expected by Eqs. (6) and (9), respectively. The reason for this are again slight deviations 

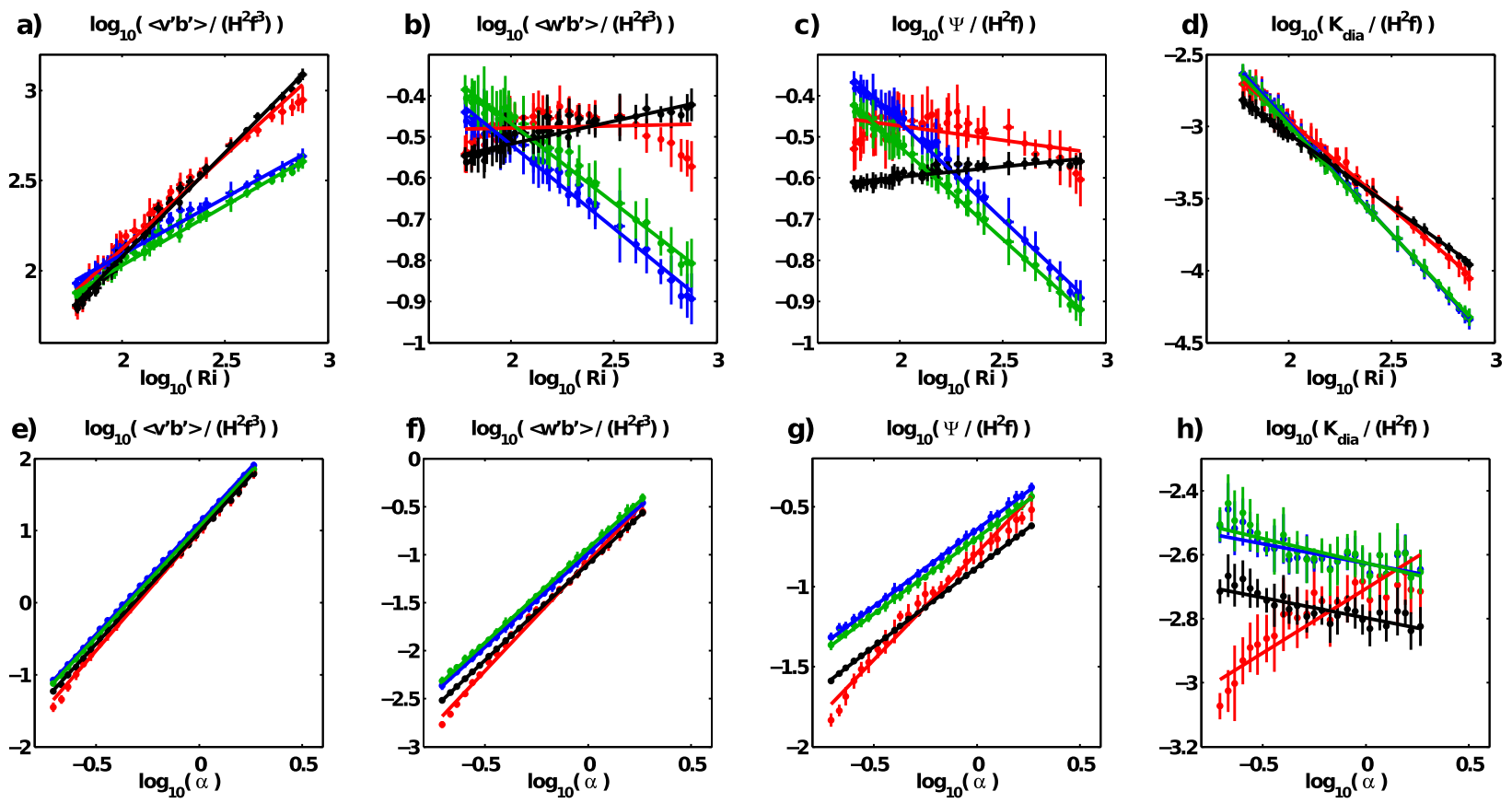

FIG. 11. Dependency of the horizontal and vertical eddy fluxes (a),(d) $\overline{v^{\prime} b^{\prime}}$ and (b),(e) $\overline{w^{\prime} b^{\prime}}$, (c),(f) eddy streamfunction $\Psi$ and (d),(g) diapycnal diffusivity $K_{\mathrm{dia}}$ on Ri for $\alpha_{0}=4$ in (a)-(d) and on $\alpha$ for $\mathrm{Ri}_{0}=1$ in (e)-(g) in the spindown scenario. Red dots denote the ensemble mean of the diagnosed variables, blue of the NLS, green of the ALS, and black of the PER parameterization, and crosses indicate the standard deviation of the ensemble spread. Each ensemble consists of 10 simulations that deviate only in small random initial perturbations. Straight lines are a least squares linear fit as detailed in the text. The slopes of these fits in the double logarithmic plots indicate the leading-order dependency on Ri for (a)-(d) and on $\alpha$ for (e)-(h) (values are given in Table 2).

in $\alpha$ throughout the experiments shown in Figs. 11a-d and slight variations in $\mathrm{Ri}$ within the experiments shown in Figs. 11e-h.

In the spindown scenario, PER tends to better describe the diagnosed eddy fluxes in comparison to NLS and ALS. The slopes that indicate the leading-order dependency on $\mathrm{Ri}$ and $\alpha$ and that are determined by the linear regressions in Fig. 11 are given in Table 5. For instance, $\overline{v^{\prime} b^{\prime}}$ determined by PER matches quite well with the diagnosed $\overline{v^{\prime} b^{\prime}}$ for the experiments with varying $\mathrm{Ri}_{0}$ (Fig. 11a). Note that the $\mathrm{Ri}$ dependency estimated here quite well agrees with findings from Bachman and Fox-Kemper (2013). However, the decrease of $\overline{w^{\prime} b^{\prime}}$ and $\Psi$ for larger Ri in Figs. 11b and 11c, respectively, is not captured by PER. On the other hand, it is too strong in ALS and NLS. Although Fig. 11d suggests that $K_{\text {dia }}$ is better represented by ALS and NLS, the diagnosed slope of the Ri dependency is better matched by PER. As in the equilibrated scenario, the simulations with fixed $\mathrm{Ri}_{0}$ and varying $\alpha_{0}$ shown in Figs. 11e-h indicate that both parameterizations predict the correct dependency on $\alpha$ for $\overline{v^{\prime} b^{\prime}}$ and $\overline{w^{\prime} b^{\prime}}$. Deviations occur only between parameterized and diagnosed $\Psi$ and $K_{\text {dia }}$, since all parameterizations seem to overestimate $\Psi$ and $K_{\text {dia }}$.
In Fig. 12, we show the vertical structure of $\overline{v^{\prime} b^{\prime}}, \overline{w^{\prime} b^{\prime}}$, $\Psi$, and $K_{\text {dia }}$ normalized by the corresponding maximum value of each profile. Note that again only the globalmean values of $M^{2}$ and $N^{2}$ enter the calculations of $\overline{v^{\prime} b^{\prime}}$ and $\overline{w^{\prime} b^{\prime}}$ for the PER and ALS parameterization, while the zonally and meridionally averaged profiles of $M^{2}$ and $N^{2}$ enter the calculations of $\Psi$ and $K_{\mathrm{dia}}$ and also the calculation of $\overline{v^{\prime} b^{\prime}}$ and $\overline{w^{\prime} b^{\prime}}$ for NLS. The meridional eddy flux $\overline{v^{\prime} b^{\prime}}$ decreases slightly at the top and at the bottom, but the decrease is not as strong as suggested by the parabolic structure function for $\overline{v^{\prime} b^{\prime}}$ by PER. There is a better agreement between the constant profile suggested by NLS and ALS and the diagnosed profile in the spindown scenario, especially for larger Ri. On the other hand, the diagnosed profile for $\overline{w^{\prime} b^{\prime}}$ is again well

TABLE 5. Exponents $\kappa$ and $\lambda$ of the estimated $\mathrm{Ri}^{\kappa}$ and $\alpha^{\lambda}$ dependency of the eddy fluxes, streamfunction, and diapycnal diffusivity in the spindown scenario.

\begin{tabular}{lrrrrrrrr}
\hline & $\kappa_{\text {diag }}$ & $\kappa_{\text {PER }}$ & $\kappa_{\mathrm{NLS}}$ & $\kappa_{\text {ALS }}$ & $\lambda_{\text {diag }}$ & $\lambda_{\text {PER }}$ & $\lambda_{\text {NLS }}$ & $\lambda_{\text {ALS }}$ \\
$\overline{\overline{v^{\prime} b^{\prime}}}$ & 1.0 & 1.2 & 0.6 & 0.7 & 3.3 & 3.1 & 3.1 & 3.1 \\
$\overline{w^{\prime} b^{\prime}}$ & -0.0 & 0.1 & -0.4 & -0.4 & 2.3 & 2.0 & 1.9 & 2.0 \\
$\Psi$ & -0.1 & 0.1 & -0.5 & -0.5 & 1.3 & 1.0 & 1.0 & 0.9 \\
$K_{\text {dia }}$ & -1.2 & -1.0 & -1.6 & -1.5 & 0.4 & -0.1 & -0.1 & -0.2 \\
\hline
\end{tabular}



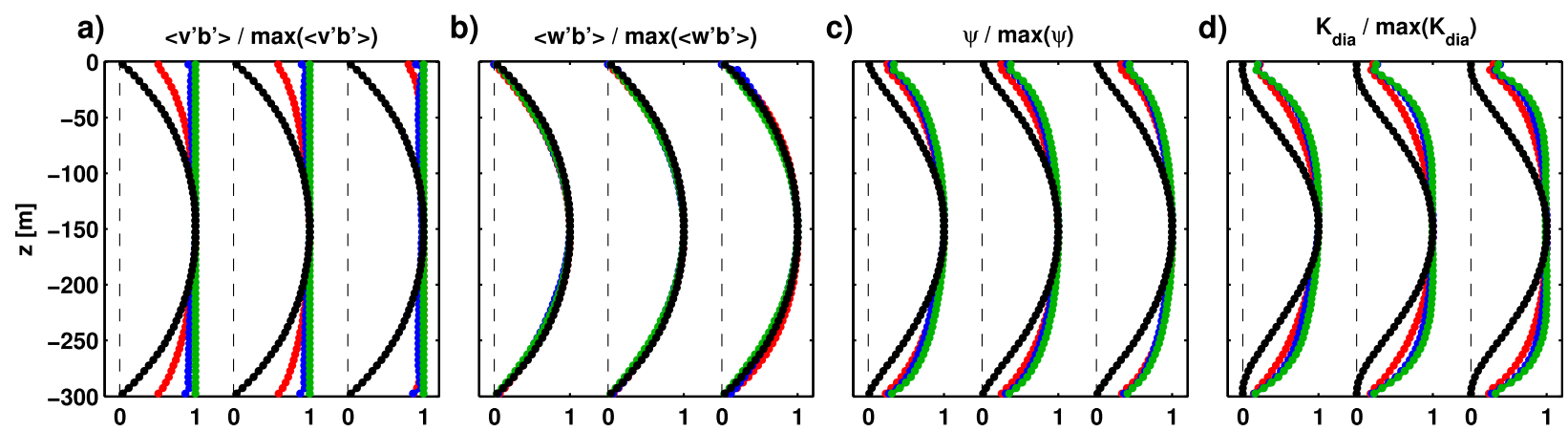

FIG. 12. Vertical profiles for (a) $\overline{v^{\prime} b^{\prime}}$, (b) $\overline{w^{\prime} b^{\prime}}$, (c) $\Psi$, and (d) $K_{\mathrm{dia}}$ for the diagnosed eddy fluxes in red and the PER, NLS, and ALS parameterizations in black, blue, and green, respectively. All profiles are normalized by their maximum value, while the dashed black lines indicate the respective zero lines. The three experiments are all performed with $\alpha_{0}=4$, but with $\mathrm{Ri}_{0}$ varying from left to right as $\mathrm{Ri}_{0}=1$, $\mathrm{Ri}_{0}=10$, and $\mathrm{Ri}_{0}=160$.

matched by all parameterizations. The diagnosed profiles of $\Psi$ and $K_{\mathrm{dia}}$ are better matched by NLS and ALS than by PER.

\section{c. Sensitivity simulations in the spindown scenario}

Although diabatic and dissipative effects are reduced in the spindown scenario, they do not vanish due to the implicit numerical mixing inherent to the advection scheme we use and the explicit harmonic friction. To investigate how the $\mathrm{Ri}$ dependencies of the eddy fluxes depend on these effects, we perform sensitivity experiments in which we switch between different dissipation and mixing schemes and vary the corresponding viscosities and diffusivities.

In one set of experiments, we half (LS) and double (HS) the Smagorinsky coefficient $C_{\text {Smag }}$ in comparison to STD. In another set of experiments, we use biharmonic friction with different viscosities instead of the harmonic Smagorinsky friction (experiments ALK3, AIK3, and AHK3). Different strengths of the diffusive effects are simulated by using biharmonic diffusion with different diffusivities $K_{4}$ together with biharmonic friction (experiments AIKL, AIKI, and AIKH). In these experiments we use a centered, second-order advection scheme instead of the third-order upwind scheme. Additionally, we perform a set of experiments with a 4 times increased horizontal and a doubled vertical resolution (H4V2). A detailed overview of the parameters used for this sensitivity study can be found in Table 6 .

As can be inferred from Fig. 13, the sensitivity experiments in the spindown simulations show a similar behavior as in the forced dissipative scenario: the less dissipative and diffusive the simulations are, the stronger are the eddy fluxes. While we find that nearly all simulations follow the $\mathrm{Ri}$ dependency of PER of $\overline{v^{\prime} b^{\prime}} \propto \mathrm{Ri}^{1}$ and $\overline{w^{\prime} b^{\prime}} \propto \mathrm{Ri}^{0}$, as also found by Bachman and Fox-Kemper (2013), the magnitude of the proportionality constant $C_{F}$ varies about $30 \%$, as can be inferred from Table 7. Although the scaling of PER seems better suited than that of ALS to capture the Ri dependency of the eddy fluxes in the spindown scenario, uncertainties arise due to the variations of the proportionality constant $C_{F}$. For very diffusive and dissipative simulations (AHK3 and AIKH), we diagnose values of $C_{F} \approx 0.06$, close to what Fox-Kemper et al. (2008) find from their simulations. For simulations with small diffusion and dissipation (H4V2, LS, ALK3, and AIKL), we find values of $C_{F} \approx$ 0.09 . Therefore, we conclude that the strength of the eddy fluxes depends much stronger on the strength of the mixing and dissipation than on the resolution (note that H4V2 has a 4 times increased horizontal and a doubled vertical resolution in comparison to the other experiments).

Note that the vertical structure of the eddy fluxes shows hardly any sensitivities to the different sensitivity experiments (not shown). In particular, $\overline{v^{\prime} b^{\prime}}$ is constant

TABLE 6. Parameters for different sensitivity experiments in the forced dissipative scenario. Note that a third-order upwind advection scheme is applied in all simulations where the biharmonic diffusivity $K_{4}$ is zero. In all other simulations we use a second-order scheme with central differences that does not produce any numerical diffusion.

\begin{tabular}{llcccc}
\hline \hline Expt & $n_{x} \times n_{z}$ & $k_{\text {max }, 0} d x$ & $C_{\text {Smag }}$ & $U_{0} d x^{3} / A_{4}$ & $U_{0} d x^{3} / K_{4}$ \\
\hline STD & $128 \times 60$ & 0.63 & 1 & $\infty$ & $\infty$ \\
H4V2 & $512 \times 120$ & 0.16 & 1 & $\infty$ & $\infty$ \\
LS & $128 \times 60$ & 0.63 & 0.5 & $\infty$ & $\infty$ \\
HS & $128 \times 60$ & 0.63 & 2 & $\infty$ & $\infty$ \\
ALK3 & $128 \times 60$ & 0.63 & 0 & 100 & $\infty$ \\
AIK3 & $128 \times 60$ & 0.63 & 0 & 10 & $\infty$ \\
AHK3 & $128 \times 60$ & 0.63 & 0 & 1 & $\infty$ \\
AIKL & $128 \times 60$ & 0.63 & 0 & 10 & 20 \\
AIKI & $128 \times 60$ & 0.63 & 0 & 10 & 10 \\
AIKH & $128 \times 60$ & 0.63 & 0 & 10 & 1 \\
\hline
\end{tabular}


a) $\log _{10}\left(<v^{\prime} b^{\prime}>/\left(H^{2} f^{3} \alpha^{3}\right)\right)$

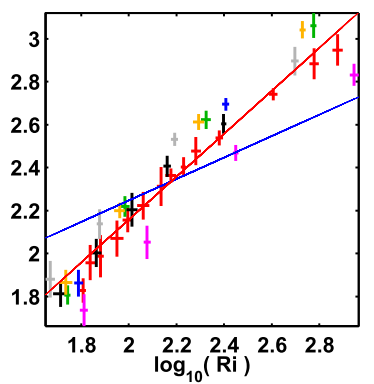

b) $\log _{10}\left(<w^{\prime} b^{\prime}>/\left(H^{2} f^{3} \alpha^{2}\right)\right)$

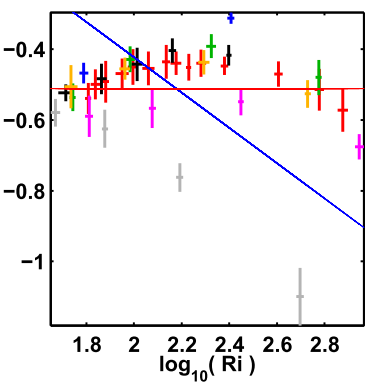

c) $\log _{10}\left(\psi /\left(\mathrm{H}^{2} \mathrm{f} \alpha\right)\right)$

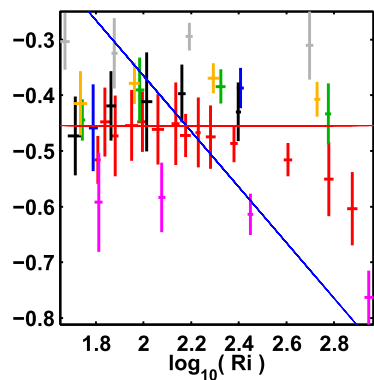

d) $\quad \log _{10}\left(K_{\text {dia }} /\left(\mathrm{H}^{2} \mathrm{f}\right)\right)$

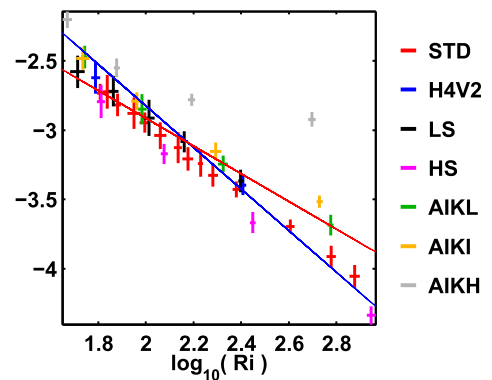

FIG. 13. Ri dependency for the sensitivity experiments listed in Table 6 for (a) $\overline{v^{\prime} b^{\prime}}$, (b) $\overline{w^{\prime} b^{\prime}}$, (c) $\Psi$, and (d) $K_{\text {dia. The colors associated }}$ with the different experiments are depicted in the legend. Solid red lines indicate the slopes with respect to PER and solid blue lines are with respect to ALS. Note that we have only plotted every third experiment for STD, and note also that we have not plotted results from the experiments AHK3, AIK3, and ALK3.

over the whole depth range for large $\mathrm{Ri}$. For smaller $\mathrm{Ri}$, it is constant in the interior and slightly decays at the surface and the bottom as shown in Fig. 12, but the decay is never stronger than $50 \%$. Therefore, the constant structure function for $\overline{v^{\prime} b^{\prime}}$ suggested from ALS better matches the diagnosed profile than the parabolic structure function suggested by PER.

\section{Summary and discussion}

Mixed layer eddies play an important role in influencing, for example, air-sea gas exchange, surface heat and freshwater fluxes, mixed layer depth, and thus biogeochemical cycles. The spatial scales of these eddies and the related mixing processes range from $100 \mathrm{~m}$ to $10 \mathrm{~km}$ and thus are too small to be resolved by current ocean models. Without accurate parameterization for mixed layer eddies, these models might therefore show a bias. Large velocity shear and low stratification are typical of the dynamics within the mixed layer featuring Richardson and Rossby numbers of order one.
Therefore, the flow is not in quasigeostrophic balance anymore and parameterizations developed for interior quasigeostrophic dynamics have to be modified.

Stone (1972a), Killworth (1997), and Eden (2011) suggest a parameterization for baroclinic instabilities based on linear stability analysis. Fox-Kemper et al. (2008), however, suggest a competing approach based on a scaling of potential energy release by eddies. The main contradiction between the two approaches lies in a different dependency of the eddy fluxes on background conditions characterized by the Richardson number Ri. Since large variations of $\mathrm{Ri}$ occur within the mixed layer, this contradiction can lead to large differences of the predicted eddy fluxes between the two parameterizations. Another difference between both parameterizations is the vertical structure of the meridional eddy flux. While the approach by Fox-Kemper et al. (2008) implies a parabolic profile, linear stability analysis suggests a constant vertical profile (Stone 1972b).

Fox-Kemper et al. (2008) compare these parameterizations with eddy fluxes diagnosed from numerical

TABLE 7. Estimated functional relationship of $\overline{v^{\prime} b^{\prime}}$ and $\overline{w^{\prime} b^{\prime}}$ on $\mathrm{Ri}$ and proportionality constants $C_{S}$ and $C_{F}$ estimated for the respective sensitivity experiments listed in Table 6 and shown in Fig. 13. Since we normalize the eddy fluxes by the respective $\alpha$ dependency, the exponents that indicate the Ri dependency for STD slightly differ from those that can be found in Table 5. We use the standard deviation of the ensemble spread to estimate the uncertainties of the depicted values.

\begin{tabular}{|c|c|c|c|c|}
\hline Expt & $\overline{v^{\prime} b^{\prime}} /\left(H^{2} f^{3} \alpha^{3}\right)$ & $\overline{w^{\prime} b^{\prime}} /\left(H^{2} f^{3} \alpha^{2}\right)$ & $C_{S}$ & $C_{F}$ \\
\hline STD & $(1.09 \pm 0.13) \mathrm{Ri}^{1.04} \pm 0.02$ & $(0.32 \pm 0.03) \mathrm{Ri}^{0.01} \pm 0.02$ & $1.16 \pm 0.02$ & $0.083 \pm 0.002$ \\
\hline $\mathrm{H} 4 \mathrm{~V} 2$ & $(0.28 \pm 0.17) \mathrm{Ri}^{1.35} \pm 0.11$ & $(0.12 \pm 0.03) \mathrm{Ri}^{0.25} \pm 0.05$ & $1.20 \pm 0.07$ & $0.096 \pm 0.006$ \\
\hline LS & $(0.69 \pm 0.34) \mathrm{Ri}^{1.17 \pm 0.10}$ & $(0.16 \pm 0.04) \mathrm{Ri}^{0.17 \pm 0.05}$ & $1.10 \pm 0.06$ & $0.094 \pm 0.005$ \\
\hline HS & $(1.28 \pm 0.53) \mathrm{Ri}^{0.94 \pm 0.07}$ & $(0.44 \pm 0.14) \mathrm{Ri}^{-0.10 \pm 0.05}$ & $1.01 \pm 0.06$ & $0.062 \pm 0.004$ \\
\hline ALK3 & $(1.83 \pm 0.65) \mathrm{Ri}^{0.97 \pm 0.07}$ & $(0.49 \pm 0.13) \mathrm{Ri}^{-0.05 \pm 0.05}$ & $1.35 \pm 0.08$ & $0.090 \pm 0.005$ \\
\hline AIK3 & $(2.26 \pm 0.72) \mathrm{Ri}^{0.95} \pm 0.06$ & $(0.65 \pm 0.13) \mathrm{Ri}^{-0.11 \pm 0.04}$ & $1.27 \pm 0.07$ & $0.085 \pm 0.005$ \\
\hline AHK3 & $(1.33 \pm 0.64) \mathrm{Ri}^{0.97} \pm 0.09$ & $(0.37 \pm 0.15) \mathrm{Ri}^{-0.07 \pm 0.08}$ & $0.91 \pm 0.09$ & $0.062 \pm 0.006$ \\
\hline AIKL & $(0.54 \pm 0.18) \mathrm{Ri}^{1.22} \pm 0.07$ & $(0.25 \pm 0.07) \mathrm{Ri}^{0.07 \pm 0.06}$ & $1.28 \pm 0.07$ & $0.089 \pm 0.005$ \\
\hline AIKI & $(0.87 \pm 0.27) \mathrm{Ri}^{1.15} \pm 0.06$ & $(0.44 \pm 0.13) \mathrm{Ri}^{-0.05 \pm 0.06}$ & $1.24 \pm 0.006$ & $0.089 \pm 0.004$ \\
\hline AIKH & $(2.19 \pm 1.02) \mathrm{Ri}^{0.98} \pm 0.09$ & $(1.63 \pm 0.58) \mathrm{Ri}^{-0.46 \pm 0.08}$ & $1.05 \pm 0.08$ & $0.079 \pm 0.006$ \\
\hline
\end{tabular}


simulations of a restratifying density front. However, they focus only on the adiabatic component of the eddy fluxes in a restratifying density front. This study aims to clarify to which extent diabatic effects of the mixed layer eddies can also be represented by the parameterizations. We consider thus modifications of the parameterizations of Fox-Kemper et al. (2008) and Stone (1972b) to also account for diabatic effects.

To evaluate the ability of the parameterizations to reproduce the eddy fluxes, we use idealized eddypermitting simulations. These simulations are performed for two different configurations, which consist of a diabatic, forced dissipative scenario and a scenario of a restratifying density front [similar to that investigated by Fox-Kemper et al. (2008) and Bachman and Fox-Kemper (2013)]. In each configuration, we run experiments for a large range of $\mathrm{Ri}$ to simulate ageostrophic and geostrophic dynamics and to estimate the Ri dependency of the eddy fluxes across these dynamical regimes. This diagnosed Ri dependency is compared to both parameterizations in order to evaluate to which extent the parameterizations are able to reproduce the simulated eddy fluxes.

In general, we find that PER is better suited to capture the magnitude of the eddy fluxes in the spindown scenario than ALS. Therefore, we confirm results by FoxKemper et al. (2008) and Bachman and Fox-Kemper (2013). The largest deviations from PER can be found for simulations with large mixing and dissipation. Similar findings result from simulations in the forced dissipative scenario. For these simulations with stronger diabatic effects, the estimated Ri dependency is in between what is proposed by PER and ALS but still closer to PER if sensitivity experiments with reduced mixing and dissipation are taken into account. An important parameter that influences the Ri dependency is the linear drag. Simulations with a reduced linear drag are closer to PER than to ALS. Additionally, we find that the eddy fluxes proposed by PER better match in simulations where we apply a zonally averaged linear drag than those proposed by ALS.

We note that estimates of the proportionality constants $C_{F}$ and $C_{S}$ of PER and ALS strongly depend on the strength of the diabatic effects. For simulations with reduced mixing and dissipation, estimated values of $C_{F}$ and $C_{S}$ are in general larger. Within the spindown scenario, we find variations of about $30 \%$. In the diabatic, forced dissipative scenario, $C_{S}$ and $C_{F}$ vary by a factor of 3 and are larger than in the spindown scenario. In the simulations with the applied zonally averaged linear drag, $C_{S}$ and $C_{F}$ are even one order of magnitude larger than in simulations with the standard linear drag.
In any case, PER does not predict the correct vertical structure of the horizontal eddy fluxes $\overline{v^{\prime} b^{\prime}}$. The diagnosed profiles of $\overline{v^{\prime} b^{\prime}}$ in both scenarios suggest a constant profile rather than a parabolic-shaped one predicted by PER. Therefore, we suggest combining both parameterizations in the sense that the magnitude is chosen as suggested by PER but that the vertical structure functions are taken from ALS.

Considering the eddy fluxes cast in an eddy streamfunction $\Psi$ and a diapycnal diffusivity $K_{\text {dia }}$, we find values for $\Psi$ on the order of $10 \mathrm{~m}^{2} \mathrm{~s}^{-1}$ and $K_{\text {dia }}$ on the order of $0.1 \mathrm{~m}^{2} \mathrm{~s}^{-1}$ for the smallest Ri and values for $\Psi$ on the order of $0.1 \mathrm{~m}^{2} \mathrm{~s}^{-1}$ and $K_{\mathrm{dia}}$ on the order of $1 \times$ $10^{-5} \mathrm{~m}^{2} \mathrm{~s}^{-1}$ for the largest Ri. Thus, the adiabatic part of the dynamics dominates the diabatic part that is represented in our model by the idealized buoyancy forcing and numerical mixing. This is also true for small $\mathrm{Ri}$, where the dynamics are more diabatic than for larger Ri.

Within this study, we make some assumptions to simplify the diagnostics. There is no consideration of a change of planetary vorticity ( $\beta$ effect, where $\beta$ denotes the meridional gradient of the Coriolis parameter f). An influence of $\beta$ on mesoscale eddies can be expected as soon as the Rhines scale $L_{\beta}=\sqrt{2 U_{\text {rms }} / \beta}$ becomes smaller than the Rossby radius $L_{r}=N H / f$ (e.g., Eden 2007). If we approximate the root-mean-square velocity $U_{\text {rms }}$ by the thermal wind, the ratio between the Rhines scale and Rossby radius can be expressed as $L_{r} / L_{\beta}=\sqrt{\alpha \operatorname{Ri} H \beta / f}$. Even for the largest $\mathrm{Ri}$ and $\alpha$ considered here, this ratio is small for midlatitude values of $f$ and $\beta$ and for a water depth $H$ appropriate for the mixed layer. Therefore, we do not expect changes in the planetary vorticity to be relevant for eddy fluxes in the mixed layer at midlatitudes. For interior dynamics, with large $H$ and probably larger Ri, however, it is reasonable to assume that effects by the planetary vorticity gradient become important and we refer to Eden (2011) and Eden (2012) where linear stability analysis was successfully used to parameterize eddy fluxes for quasigeostrophic flows including the $\beta$ effect.

Another simplification we make in this study is to consider the mixed layer isolated from the abyssal ocean and to apply a solid bottom at the mixed layer base. However, we do not expect large influences of the abyssal circulation on the mixed layer eddy fluxes as long as the increase of $N^{2}$ within the pycnocline is large and changes in the vertical shear of the horizontal velocity are small. In these cases, the mixed layer and the interior ocean can be considered as separated regimes. Thomsen et al. (2014) show for a typical situation of a boundary current that NLS suggests two maxima of the growth rate: one corresponding to an interior mode and the other one to the mixed layer mode considered within 
this study. The eddy fluxes of the mixed layer mode quickly vanish below the mixed layer base as also observed in numerical model studies of Fox-Kemper and Ferrari (2008). However, Badin et al. (2011) show that there can be an influence of mixed layer eddies on lateral tracer mixing within the pycnocline. To account for interactions between the mixed layer and the pycnocline, both parameterizations considered in this study have to be modified. For the parameterization based on linear stability analysis, this would mean to derive analytical approximations for more complicated profiles of $N^{2}$ and $M^{2}$ as in Killworth (1997) and Eden (2012).

Neither of the parameterizations accounts for horizontal changes of $N^{2}$ and $M^{2}$. As long as these variations occur on scales larger than the mixed layer Rossby radius, the eddy fluxes might be calculated with the varying $N^{2}$ and $M^{2}$ in a Wentzel-Kramers-Brillouin (WKB) sense. For variations of $N^{2}$ and $M^{2}$ on scales at or below the mixed layer Rossby radius, it is not clear whether the parameterizations yield reasonable results, since lateral shear instabilities might have other characteristics than baroclinic instability. Furthermore, we have not accounted for nonhydrostatic effects. Guidance of how these effects might be implemented in ALS can be drawn from Stone (1971). According to Mahadevan (2006), who does not find major differences between a nonhydrostatic and hydrostatic spindown simulation for parameters similar to those applied here, and in accordance to Stone (1971), who reports that nonhydrostatic effects become relevant only for $\alpha \ll 1$, we do not expect these effects to play an important role for the parameter range considered in this study. Since the thermal wind relation is a basic ingredient to determine the eddy velocity scale, both parameterizations are also not likely to make accurate predictions at the equator. On the other hand, it might be possible to extend both parameterizations to regions at the equator by replacing $|f|$ with $\sqrt{f^{2}+\tau^{-2}}$ (Young 1994; Eden 2006; Fox-Kemper et al. 2011).

Acknowledgments. This work was supported by BMBF-SOPRAN FKZ 03F0662E. We are thankful for comments by Baylor Fox-Kemper and an anonymous reviewer that improved this manuscript.

\section{APPENDIX A}

\section{Nondimensionalized Equations of Motion}

We nondimensionalize the inviscid, adiabatic, NavierStokes equations in Boussinesq approximation to identify important characteristic parameters. By using the following scales as in, for example, Stone (1970),

$$
\begin{aligned}
t & =\frac{L_{0}}{U_{0}} \hat{t}, \quad f=f_{0}, \\
(x, y) & =L_{0}(\hat{x}, \hat{y}), \quad z=H_{0} \hat{z}, \\
\partial_{z} b & =N_{0}^{2} \partial_{z} \hat{b}, \quad \partial_{y} b=M_{0}^{2} \partial_{y} \hat{b} \\
p & =N_{0}^{2} H_{0}^{2} \hat{p}, \quad b=N_{0}^{2} H_{0} \hat{b}, \quad \text { and } \\
(u, v) & =U_{0}(\hat{u}, \hat{v}), \quad w=H_{0} f_{0} \hat{w},
\end{aligned}
$$

where dimensionless variables are denoted by hats, we obtain

$$
\begin{aligned}
\operatorname{Ro} D_{\hat{t}} \hat{u}-\hat{v} & =-\operatorname{RoRi}_{\hat{x}} \hat{p}, \\
\operatorname{Ro} D_{\hat{t}} \hat{v}+\hat{u} & =-\operatorname{RoRi}_{\hat{y}} \hat{p}, \\
\delta^{2} D_{\hat{t}} \hat{w} & =-\operatorname{Ri}\left(\partial_{\hat{z}} \hat{p}-\hat{b}\right), \\
\partial_{\hat{x}} \hat{u}+\partial_{\hat{y}} \hat{v}+\partial_{\hat{z}} \hat{w} & =0, \text { and } \\
D_{\hat{t}} \hat{b} & =0 .
\end{aligned}
$$

This set of equations contains three parameters, namely, the aspect ratio $\delta=H_{0} / L_{0}$, the Rossby number Ro $=U_{0} /\left(L_{0} f_{0}\right)$, and the Richardson number $\mathrm{Ri}=N_{0}^{2} H_{0}^{2} / U_{0}^{2}$. While the magnitude of $\delta$ determines if nonhydrostatic effects are important, the magnitude of $\mathrm{Ri}$ and Ro determines to which extent ageostrophic effects have to be considered.

Note that a slightly different scaling $w=f_{0} U_{0}^{2} /\left(N_{0}^{2} H_{0}\right) \hat{w}$ and $p=f U_{0} L_{0} \hat{p}$ was proposed by McWilliams (1985) and Molemaker et al. (2005). Although this scaling yields a different weighting between the single terms in the Navier-Stokes equations, the resulting set of equations is still sufficiently described by Ri, Ro, and $\delta$.

The background flow determines only two parameters $\mathrm{Ri}$ and $\alpha=\mathrm{Ro} / \delta$. To evaluate the magnitude of the single terms in Eqs. (A6)-(A10), an additional assumption on, for example, the length scale $L_{0}$ is necessary. Three different assumptions for $L_{0}$ are made by different authors:

(i) $L_{0}=N_{0} H_{0} / f_{0}=\sqrt{\mathrm{Ri}}(\mathrm{Ro} / \delta) H_{0}$, that is, $L_{0}$ is chosen to be the Rossby radius (Molemaker et al. 2005), leading to

$$
\delta=\frac{f_{0}}{N_{0}}, \quad \mathrm{Ro}=\frac{M_{0}^{2}}{f_{0} N_{0}}, \quad \mathrm{Ro}^{2} \mathrm{Ri}=1 .
$$

(ii) $L_{0}=U_{0} / f_{0}=(\mathrm{Ro} / \delta) H_{0}($ Stone 1970), leading to

$$
\delta=\frac{f_{0}^{2}}{M_{0}^{2}}, \quad \text { Ro }=1 .
$$


(iii) $L_{0}=\left(N_{0}^{2} / M_{0}^{2}\right) H_{0}=\operatorname{Ri}(\mathrm{Ro} / \delta) H_{0}($ Fox-Kemper et al. 2008), leading to

$$
\delta=\frac{M_{0}^{2}}{N_{0}^{2}}, \quad \text { Ro }=\frac{M_{0}^{4}}{N_{0}^{2} f_{0}^{2}}, \quad \text { RiRo }=1 .
$$

Each of these assumptions relates the aspect ratio to the characteristic properties of the background flow $N_{0}^{2}, M_{0}^{2}$, and $f_{0}$, and it reduces the number of the characteristic parameters of the problem. Since we only consider $\alpha=\mathrm{Ro} / \delta$, these different scalings have no direct influence on the parameterizations Eqs. (6) and (9) or the prescribed initial conditions of our numerical simulations. However, for identifying which terms in Eqs. (A6)-(A10) are relevant for a certain background state, one of the above choices for $L_{0}$ has to be made in order to relate Ro and $\delta$ separately to this background state.

\section{APPENDIX B}

\section{Eddy Fluxes in the Eady Problem}

We linearize the quasigeostrophic potential vorticity equation to obtain

$$
\left(\partial_{t}+U \partial_{x}\right)\left(\nabla_{h}^{2} \psi^{\prime}+\frac{f^{2}}{N^{2}} \partial_{z z} \psi^{\prime}\right)=0
$$

where $\psi^{\prime}=\bar{\psi}-\psi$ is the perturbation of the horizontal streamfunction $\psi$ with respect to the streamfunction of the background flow, which is given by $\bar{\psi}=-U_{0}(z / H+1) y$ for a zonal flow in thermal wind balance $U=U_{0}(z / H+1)$ with amplitude $U_{0}=-M^{2} H / f$, where $M^{2}$ denotes a constant meridional buoyancy gradient, $H$ is the water depth, and $f$ is the Coriolis parameter. Using a wave ansatz $\psi^{\prime}=\phi(z) e^{i(\omega t-k x-l y)}$, we obtain a differential equation for $\phi(z):$

$$
\phi-\frac{H^{2}}{L_{r}^{2} k_{h}^{2}} \partial_{z z} \phi=0
$$

where $k_{h}=\left(k^{2}+l^{2}\right)^{-1 / 2}$ denotes the horizontal wavenumber. Equation (B2) has the solution $\phi=$ $A \cosh \left(L_{r} k_{h} z / H\right)+B \sinh \left(L_{r} k_{h} z / H\right)$. The vertical velocity $w$ is derived from $\psi$ by $w=-\left(f / N^{2}\right) D_{t} \partial_{z} \psi$ that reads in the linearized form

$$
w=-\frac{f}{N^{2}}\left[(i \omega-i U k) \partial_{z} \phi+i \frac{U_{0}}{H} k \phi\right] .
$$

Rigid-lid boundary conditions $w=0$ at $z=0$ and $z=$ $-H$ yield

$$
\begin{aligned}
A & =\frac{U_{0} k-\omega}{U_{0} k} \kappa B, \\
\frac{\omega}{U_{0} k} & =\frac{1}{2} \pm i \frac{F(\kappa)}{\kappa},
\end{aligned}
$$

with $F(\kappa)=\sqrt{\kappa \operatorname{coth}(\kappa)-\kappa^{2} / 4-1}$ and $\kappa=L_{r} k_{h}$. Exponential growth and therefore instability can be expected if $\omega_{i}=\operatorname{Im}\{\omega\}<0$, especially if the maximum exponential growth rate $\sigma_{\max }=\max \left(-\omega_{i}\right)$ is obtained for $k=\kappa_{1} / L_{r}$ and $l=0$ with $\kappa_{1} \approx 1.6$ and $F\left(\kappa_{1}\right) \approx 0.3$ (Eady 1949).

As soon as the perturbations reach finite amplitude, the exponential growth is inhibited. We assume that this happens if $v^{\prime} \propto \sigma_{\max } / k_{\max }$ (Killworth 1997). Therefore, we obtain $B=C \sigma_{\max } / k_{\max }^{2}$ with a tuning constant $C$ of order one. Hence, we can calculate the meridional eddy fluxes $\overline{v^{\prime} b^{\prime}}$,

$\overline{v^{\prime} b^{\prime}}=\frac{1}{2} \operatorname{Re}\left\{-i f k \phi \partial_{z} \phi^{*}\right\}=\frac{C^{2}}{2} \frac{F(\kappa)^{3}}{\kappa^{2}} \frac{M^{4} H^{2} N}{f^{2}}$,

and the vertical eddy fluxes $\overline{w^{\prime} b^{\prime}}$,

$$
\begin{aligned}
\overline{w^{\prime} b^{\prime}} & =\frac{f^{2}}{2 N^{2}} \operatorname{Re}\left\{-i\left[(\omega-U k)\left|\partial_{z} \phi\right|^{2}+\frac{k U_{0}}{H} \phi \partial_{z} \phi^{*}\right]\right\} \\
& =-\frac{C^{2}}{4} \frac{F(\kappa)^{3}}{\kappa} \tanh \left(\frac{\kappa}{2}\right) \frac{M^{6} H^{2}}{N^{3} f^{2}} \mu_{E}(z),
\end{aligned}
$$

with the structure function $\mu_{E}(z)$ that peaks at one and for which we obtain

$$
\mu_{E}(z)=\frac{\cosh \left[\kappa\left(\frac{2 z}{H}+1\right)\right]-\cosh (\kappa)}{1-\cosh (\kappa)} .
$$

Note that for $z=-H / 2$ and for the maximum growth rate with $\kappa=\kappa_{1} \approx 1.6$, the eddy flux ratio is $\overline{w^{\prime} b^{\prime}} / \overline{v^{\prime} b^{\prime}}=$ $-\left(\kappa_{1} / 2\right) \tanh \left(\kappa_{1} / 2\right) M^{2} / N^{2} \approx-0.53 M^{2} / N^{2}$.

\section{REFERENCES}

Bachman, S., and B. Fox-Kemper, 2013: Eddy parameterization challenge suite I: Eady spindown. Ocean Modell., 64, 12-28, doi:10.1016/j.ocemod.2012.12.003.

Badin, G., A. Tandon, and A. Mahadevan, 2011: Lateral mixing in the pycnocline by baroclinic mixed layer eddies. J. Phys. Oceanogr., 41, 2080-2101, doi:10.1175/JPO-D-11-05.1.

Boccaletti, G., R. Ferrari, and B. Fox-Kemper, 2007: Mixed layer instabilities and restratification. J. Phys. Oceanogr., 37, 22282250, doi:10.1175/JPO3101.1.

Capet, X., E. J. Campos, and A. M. Paiva, 2008: Submesoscale activity over the Argentinian shelf. Geophys. Res. Lett., 35, L15605, doi:10.1029/2008GL034736.

Eady, E. T., 1949: Long waves and cyclone waves. Tellus, 1, 33-52, doi:10.1111/j.2153-3490.1949.tb01265.x. 
Eden, C., 2006: Middepth equatorial tracer tongues in a model of the Atlantic Ocean. J. Geophys. Res., 111, C12025, doi:10.1029/ 2006JC003565.

- 2007: Eddy length scales in the North Atlantic Ocean. J. Geophys. Res., 112, C06004, doi:10.1029/2006JC003901.

— 2011: A closure for meso-scale eddy fluxes based on linear instability theory. Ocean Modell., 39, 362-369, doi:10.1016/ j.ocemod.2011.05.009.

_ 2012: Implementing diffusivities from linear stability analysis in a three-dimensional general circulation ocean model. Ocean Modell., 57-58, 15-28, doi:10.1016/j.ocemod.2012.08.001.

__ , and R. J. Greatbatch, 2008a: Diapycnal mixing by mesoscale eddies. Ocean Modell., 23, 113-120, doi:10.1016/ j.ocemod.2008.04.006.

— Modell., 20, 223-239, doi:10.1016/j.ocemod.2007.09.002.

Fox-Kemper, B., and R. Ferrari, 2008: Parameterization of mixed layer eddies. Part II: Prognosis and impact. J. Phys. Oceanogr., 38, 1166-1179, doi:10.1175/2007JPO3788.1.

,$- \ldots$, and R. Hallberg, 2008: Parameterization of mixed layer eddies. Part I: Theory and diagnosis. J. Phys. Oceanogr., 38, 1145-1165, doi:10.1175/2007JPO3792.1.

_- , and Coauthors, 2011: Parameterization of mixed layer eddies. III: Implementation and impact in global ocean climate simulations. Ocean Modell., 39, 61-78, doi:10.1016/ j.ocemod.2010.09.002.

Gent, P. R., and J. C. McWilliams, 1990: Isopycnal mixing in ocean circulation models. J. Phys. Oceanogr., 20, 150-155, doi:10.1175/1520-0485(1990)020<0150:IMIOCM>2.0.CO;2.

_ J. Willebrand, T. J. McDougall, and J. C. McWilliams, 1995: Parameterizing eddy-induced tracer transports in ocean circulation models. J. Phys. Oceanogr., 25, 463-474, doi:10.1175/ 1520-0485(1995)025<0463:PEITTI > 2.0.CO;2.

Green, J. S. A., 1970: Transfer properties of the large-scale eddies and the general circulation of the atmosphere. Quart. J. Roy. Meteor. Soc., 96, 157-185, doi:10.1002/qj.49709640802.

Haine, T. W. N., and J. Marshall, 1998: Gravitational, symmetric, and baroclinic instability of the ocean mixed layer. J. Phys. Oceanogr., 28, 634-658, doi:10.1175/1520-0485(1998)028<0634: GSABIO $>2.0 . \mathrm{CO} ; 2$.

Held, I. M., and T. Schneider, 1999: The surface branch of the zonally averaged mass transport circulation in the troposphere. J. Atmos. Sci., 56, 1688-1697, doi:10.1175/ 1520-0469(1999)056<1688:TSBOTZ>2.0.CO;2.

Killworth, P., 1997: On the parameterization of eddy transfer Part I. Theory. J. Mar. Res., 55, 1171-1197, doi:10.1357/ 0022240973224102.

Klein, P., B. L. Hua, G. Lapeyre, X. Capet, S. Le Gentil, and H. Sasaki, 2008: Upper ocean turbulence from high-resolution 3D simulations. J. Phys. Oceanogr., 38, 1748-1763, doi:10.1175/ 2007JPO3773.1.

Mahadevan, A., 2006: Modeling vertical motion at ocean fronts: Are nonhydrostatic effects relevant at submesoscales? Ocean Modell., 14, 222-240, doi:10.1016/j.ocemod.2006.05.005.

Marshall, J., A. Adcroft, C. Hill, L. Perelman, and C. Heisey, 1997: A finite-volume, incompressible Navier Stokes model for studies of the ocean on parallel computers. J. Geophys. Res., 102, 5753-5766, doi:10.1029/96JC02775.

McWilliams, J. C., 1985: Submesoscale, coherent vortices in the ocean. Rev. Geophys., 23, 165-182, doi:10.1029/RG023i002p00165.

Molemaker, M. J., J. C. McWilliams, and I. Yavneh, 2005: Baroclinic instability and loss of balance. J. Phys. Oceanogr., 35, 1505-1517, doi:10.1175/JPO2770.1.

Munk, W., L. Armi, K. Fischer, and F. Zachariasen, 2000: Spirals on the sea. Proc. Roy. Soc. London, A456, 1217-1280, doi:10.1098/rspa.2000.0560.

Oschlies, A., 2002: Improved representation of upper-ocean dynamics and mixed layer depths in a model of the North Atlantic on switching from eddy-permitting to eddy-resolving grid resolution. J. Phys. Oceanogr., 32, 2277-2298, doi:10.1175/ 1520-0485(2002)032<2277:IROUOD > 2.0.CO;2.

Plumb, R. A., and R. Ferrari, 2005: Transformed Eulerian-mean theory. Part I: Nonquasigeostrophic theory for eddies on a zonal-mean flow. J. Phys. Oceanogr., 35, 165-174, doi:10.1175/ JPO-2669.1.

Smagorinsky, J., 1963: General circulation experiments with the primitive equations: I. The basic experiment. Mon. Wea. Rev., 91, 99-164, doi:10.1175/1520-0493(1963)091<0099: GCEWTP $>2.3 . \mathrm{CO} ; 2$.

Stone, P. H., 1966: On non-geostrophic baroclinic stability. J. Atmos. Sci., 23, 390-400, doi:10.1175/1520-0469(1966)023<0390: ONGBS $>2.0 . \mathrm{CO} ; 2$.

, 1970: On non-geostrophic baroclinic stability: Part II. J. Atmos. Sci., 27, 721-726, doi:10.1175/1520-0469(1970)027<0721: ONGBSP $>2.0 . \mathrm{CO} ; 2$.

, 1971: Baroclinic stability under non-hydrostatic conditions. J. Fluid Mech., 45, 659-671, doi:10.1017/S0022112071000260.

__, 1972a: A simplified radiative-dynamical model for the static stability of rotating atmospheres. J. Atmos. Sci., 29, 405-418, doi:10.1175/1520-0469(1972)029<0405: ASRDMF $>2.0 . \mathrm{CO} ; 2$.

__, 1972b: On non-geostrophic baroclinic stability: Part III. The momentum and heat transports. J. Atmos. Sci., 29, 419-426, doi:10.1175/1520-0469(1972)029<0419: ONGBSP $>2.0 . \mathrm{CO} ; 2$.

Tandon, A., and C. Garrett, 1996: On a recent parameterization of mesoscale eddies. J. Phys. Oceanogr., 26, 406-411, doi:10.1175/ 1520-0485(1996)026<0406:OARPOM $>2.0 . C O ; 2$.

Thompson, K. R., D. G. Wright, Y. Lu, and E. Demirov, 2006: A simple method for reducing seasonal bias and drift in eddy resolving ocean models. Ocean Modell., 13,109-125, doi:10.1016/ j.ocemod.2005.11.003.

Thomsen, S., C. Eden, and L. Czeschel, 2014: Stability analysis of the Labrador Current. J. Phys. Oceanogr., 44, 445-463, doi:10.1175/JPO-D-13-0121.1.

Treguier, A. M., I. M. Held, and V. D. Larichev, 1997: Parameterization of quasigeostrophic eddies in primitive equation ocean models. J. Phys. Oceanogr., 27, 567-580, doi:10.1175/ 1520-0485(1997)027<0567:POQEIP>2.0.CO;2.

Young, W. R., 1994: The subinertial mixed layer approximation. J. Phys. Oceanogr., 24, 1812-1826, doi:10.1175/ 1520-0485(1994)024<1812:TSMLA > 2.0.CO;2. 